\title{
Topological sectors for Ginzburg-Landau energies
}

Luís Almeida

\section{Introduction.}

\subsection{Ginzburg-Landau functionals.}

Let $\Omega$ be the annulus $\left\{x \in \mathbb{R}^{2}: 1 / 4<|x|<1\right\} \subset \mathbb{R}^{2}$. For maps $u \in H^{1}\left(\Omega, \mathbb{R}^{2}\right)=W^{1,2}\left(\Omega, \mathbb{R}^{2}\right)$ we consider the Ginzburg-Landau functional

$$
E_{\varepsilon}(u)=\frac{1}{2} \int_{\Omega}|\nabla u|^{2}+\frac{1}{4 \varepsilon^{2}} \int_{\Omega}\left(1-|u|^{2}\right)^{2},
$$

where $\varepsilon$ is a small parameter. For $\Lambda \in \mathbb{R}^{+}$we define the energy level set $E_{\varepsilon}^{\Lambda}$ as

$$
E_{\varepsilon}^{\Lambda}:=\left\{u \in H^{1}\left(\Omega, \mathbb{R}^{2}\right): E_{\varepsilon}(u)<\Lambda\right\} .
$$

One of the main purposes of this paper is to show that given $\Lambda>0$, for $\varepsilon$ small enough, $E_{\varepsilon}^{\Lambda}$ may be multiply connected. Moreover, the connected components of $E_{\varepsilon}^{\Lambda}$ may be classified by the degree of $u$ (since $u$ is not $S^{1}$-valued, we have to be careful in order to define its degree - this is the main technical problem of our work).

Functionals like $E_{\varepsilon}$ play an important role in many low temperature physics phenomena like superfluidity. We can also find closely related functionals in the theory of superconductivity and in two-dimensional Higgs models. In our work we will consider one of these superconductivity models: the gauge-covariant Ginzburg-Landau model, 
where the energy functional may be written as

$$
F_{\varepsilon}(u, A)=\frac{1}{2} \int_{\mathbb{R}^{2}}|d A|^{2}+\frac{1}{2} \int_{\Omega}\left|\nabla_{A} u\right|^{2}+\frac{1}{4 \varepsilon^{2}} \int_{\Omega}\left(1-|u|^{2}\right)^{2},
$$

where $u \in H^{1}\left(\Omega, \mathbb{R}^{2}\right)$, as before, and $A \in H^{1}\left(\mathbb{R}^{2}, \mathbb{R}^{2}\right)$ is the gauge potential one-form,

$$
A=A_{1} d x^{1}+A_{2} d x^{2} \cong\left(\begin{array}{c}
A_{1} \\
A_{2}
\end{array}\right)=\left(A_{1}, A_{2}\right) .
$$

Here, as we will often do in this paper, we used the natural identification (given by the $\mathbb{R}^{2}$ scalar product) between the one-form $A$ and the vector with the same components which we also denote by $A$. In equation (1.3) the expression $\nabla_{A} u$ denotes the covariant derivative of $u$, i.e. $\nabla_{A} u=\nabla u-\imath A u$.

This model was introduced by Ginzburg and Landau in the 50's for the study of phase transitions in superconducting materials (see the remarks on physics below).

The main feature of the functional $F_{\varepsilon}$ is its invariance under gauge transformations. For a function $\phi \in W^{2,2}\left(\mathbb{R}^{2}, \mathbb{R}\right)$, the gauge transformation associated to $\phi$ is the map $(u, A) \longmapsto\left(u_{\phi}, A_{\phi}\right)$ given by

$$
\begin{cases}u_{\phi}=\exp (\imath \phi) u, & \text { in } \Omega, \\ A_{\phi}=A+d \phi, & \text { in } \mathbb{R}^{2} .\end{cases}
$$

In this case we say that $(u, A)$ is gauge-equivalent to $\left(u_{\phi}, A_{\phi}\right)$ and we denote this by $(u, A) \sim\left(u_{\phi}, A_{\phi}\right)$. Saying that $F_{\varepsilon}$ is gauge-invariant means that

$$
F_{\varepsilon}\left(u_{\phi}, A_{\phi}\right)=F_{\varepsilon}(u, A), \quad \text { for all } \phi \in W^{2,2}\left(\mathbb{R}^{2}, \mathbb{R}\right) \text {. }
$$

This gauge-invariance follows easily from the facts that

$$
\begin{gathered}
\left(u_{\phi}, A_{\phi}\right) \in H^{1}\left(\Omega, \mathbb{R}^{2}\right) \times H^{1}\left(\mathbb{R}^{2}, \mathbb{R}^{2}\right), \quad\left|u_{\phi}\right|=|u|, \\
d A_{\phi}=d A+d d \phi=d A, \\
\nabla_{A_{\phi}} u_{\phi}=\exp (\iota \phi) \nabla_{A} u, \text { and thus }\left|\nabla_{A_{\phi}} u_{\phi}\right|=\left|\nabla_{A} u\right| .
\end{gathered}
$$

The only quantities which are significant from the physics point of view are those, like $|u|, \nabla_{A} u$ and the magnetic field $h=\star d A$, which are invariant under gauge transformations. Other important gauge-invariant 
quantities are the current $J=\left(\imath u, \nabla_{A} u\right)$ and, the one which we are more concerned about in this paper, the degree of $u$ along a smooth closed curve $\gamma$, diffeomorphic to $S^{1}$, such that $|u| \neq 0$ on $\gamma$. In integral form, this degree is given by

$$
\operatorname{deg}(u, \gamma)=\frac{1}{2 \pi} \int_{\gamma} \frac{u}{|u|} \times \partial_{\tau}\left(\frac{u}{|u|}\right) d \tau
$$

where $\tau$ denotes the unit tangent to $\gamma$.

It is easy to see that gauge-equivalence defines an equivalence relation in $H^{1}\left(\Omega, \mathbb{R}^{2}\right) \times H^{1}\left(\mathbb{R}^{2}, \mathbb{R}^{2}\right)$. A physical state of our system is associated not with an individual configuration $(u, A)$, but with a whole equivalence class $[u, A]:=\left\{(v, B) \in H^{1}\left(\Omega, \mathbb{R}^{2}\right) \times H^{1}\left(\mathbb{R}^{2}, \mathbb{R}^{2}\right)\right.$ : $(v, B) \sim(u, A)\}$. We denote the physical space by $H_{g i}=\left[H^{1}\left(\Omega, \mathbb{R}^{2}\right) \times\right.$ $\left.H^{1}\left(\mathbb{R}^{2}, \mathbb{R}^{2}\right)\right] / \sim$, and also consider $F_{\varepsilon}$ as a functional defined on $H_{g i}$.

As in the case of $E_{\varepsilon}$, we define the energy level sets of $F_{\varepsilon}$ by

$$
F_{\varepsilon}^{\Lambda}:=\left\{[v, B] \in H_{g i}: F_{\varepsilon}([v, B])<\Lambda\right\} .
$$

Since the functional $E_{\varepsilon}$ does not involve the connection, it is a little easier to deal with than the functional $F_{\varepsilon}$. Nevertheless, as we will see in our work, most of the mathematical difficulties are already encountered in the study of $E_{\varepsilon}$. In fact, after some additional technical arguments, we deduce the classification result for the components of the level sets of $F_{\varepsilon}$, from the corresponding result for $E_{\varepsilon}$. Therefore, we start by considering the functional $E_{\varepsilon}$ given by (1.1).

\subsection{Degree of a map and definition of topological sectors.}

We consider a fixed number $\Lambda>0$, and focus our attention on the level set $E_{\varepsilon}^{\Lambda}$ defined by (1.2). First, we remark that since the notion of degree we define is continuous in $W^{1,2}(\Omega) \cap E_{\varepsilon}^{\Lambda}$ and that smooth maps are dense in $W^{1,2}(\Omega)=H^{1}(\Omega)$, it suffices to consider the case where $u \in W^{1,2}(\Omega) \cap C^{\infty}$. Hence, without loss of generality, we will always assume that $u$ is smooth in this paper.

Based on the work of B. White [28] (see also the work of F. Bethuel [6]), for maps $u \in W^{1,2}\left(\Omega, S^{1}\right)$, i.e. for the case when $|u| \equiv 1$, we can define the degree of $u$ in $\Omega, \operatorname{deg}(u, \Omega)$, as the degree of the restriction of $u$ to a one-dimensional skeleton of $\Omega$ - for instance, in case $u$ is continuous, this can be any circle $S_{r}=\{x:|x|=r\}$, for $1 / 4<r<1$ 
(if $u$ is not continuous we might need to move the circle slightly in order to have a "nice" restriction). The degree can then be written, in integral form, as

$$
\operatorname{deg}(u, \Omega)=\operatorname{deg}\left(u, S_{r}\right)=\frac{1}{2 \pi} \int_{S_{r}} \frac{u}{|u|} \times \partial_{\tau}\left(\frac{u}{|u|}\right) d \tau .
$$

This definition of the degree will always give us an integer, and it classifies the homotopy classes of $W^{1,2}\left(\Omega, S^{1}\right)$. Our purpose is to extend this notion to all $u \in E_{\varepsilon}^{\Lambda}$ for $\varepsilon$ sufficiently small. In this context, our first result is given by the following Theorem.

Theorem 1. Given $\Lambda \in \mathbb{R}^{+}$, there exists $\varepsilon_{0}>0$, depending only on $\Lambda$, such that for $\varepsilon<\varepsilon_{0}$, we can define a continuous map

$$
\begin{aligned}
& \chi: E_{\varepsilon}^{\Lambda} \longrightarrow \mathbb{Z}, \\
& u \longmapsto \operatorname{deg}(u, \Omega),
\end{aligned}
$$

such that this map coincides with the classical notion of degree mentioned above when $u$ has values in $S^{1}$ (i.e. when $\left.u \in W^{1,2}\left(\Omega, S^{1}\right) \cap E_{\varepsilon}^{\Lambda}\right)$.

Usually we call the map $\chi$ the global degree in $\Omega$ and, as above, we denote $\chi(u)=\operatorname{deg}(u, \Omega)$. For each $n \in \mathbb{Z}, \chi^{-1}(n)=\left\{u \in E_{\varepsilon}^{\Lambda}\right.$ : $\operatorname{deg}(u, \Omega)=n\}$, is an open and closed subset of $E_{\varepsilon}^{\Lambda}$ which we call the $n^{\text {th }}$ topological sector of $E_{\varepsilon}^{\Lambda}$, and we also denote it by $\operatorname{top}_{n}\left(E_{\varepsilon}^{\Lambda}\right)$.

REMARK. In fact, what we prove in Theorem 1 is that the degree of $u$ is constant inside each connected component of $E_{\varepsilon}^{\Lambda}$ - we do not show that different connected components correspond to different values of the degree, which would give us a complete classification of the components by the degree of its members. We will come back to this question later on.

The asymptotic behavior, when $\varepsilon \longrightarrow 0$ of critical points of the functionals $E_{\varepsilon}$ and $F_{\varepsilon}$ was extensively studied by many authors. Among them we would like to single out the work of F. Bethuel, H. Brezis and F. Hélein [8] regarding the functional $E_{\varepsilon}$, and those of F. Bethuel and T. Rivière [9] and [10] which concern the functional $F_{\varepsilon}$.

We will give a rough description of the proof of Theorem 1 at the end of the Introduction. This proof is rather technical and will be done in sections 2 to 8 . The Euler-Lagrange equations for the functional $E_{\varepsilon}$ are called the Ginzburg-Landau equations. They can be written as

$$
-\Delta u=\frac{1}{\varepsilon^{2}} u\left(1-|u|^{2}\right), \quad \text { in } \Omega \text {. }
$$


In the context of the gauge invariant model, we can also extend the definition of degree to any configuration $[v, B] \in F_{\varepsilon}^{\Lambda}$ provided $\varepsilon$ is small enough. In fact, we prove

Theorem 2. Given $\Lambda \in \mathbb{R}^{+}$, there exists $\varepsilon_{0}>0$, depending only on $\Lambda$, such that for $\varepsilon<\varepsilon_{0}$, we can define a continuous map

$$
\begin{aligned}
& \hat{\chi}: F_{\varepsilon}^{\Lambda} \longrightarrow \mathbb{Z}, \\
& {[u, A] \longmapsto \operatorname{deg}([u, A], \Omega),}
\end{aligned}
$$

such that this map coincides with the classical notion of degree mentioned above when $u$ has values in $S^{1}$ (i.e. when $\left.u \in W^{1,2}\left(\Omega, S^{1}\right) \cap F_{\varepsilon}^{\Lambda}\right)$. Usually we call the map $\hat{\chi}$ the global degree in $\Omega$ and, as above, we denote $\hat{\chi}(u, A)=\operatorname{deg}([u, A], \Omega)$.

Minimizing $E_{\varepsilon}$ inside each component of $E_{\varepsilon}^{\Lambda}$ (or $F_{\varepsilon}$ inside each component of $F_{\varepsilon}^{\Lambda}$ ), we will obtain solutions of (1.11) which are locally minimizing, i.e. critical points of $E_{\varepsilon}$ (respectively, $F_{\varepsilon}$ ) which are local minima. These are the solutions that should be associated with permanent currents.

Moreover, we will show in the next subsection, that as a corollary of Theorems 1 and 2, we can also prove the existence of mountainpass points for $E_{\varepsilon}$ (which correspond to mountain-pass type solutions of (1.11)). An analogous reasoning gives the existence of mountainpass points for $F_{\varepsilon}$. This result is stated in Theorem 4 . Unlike the solutions obtained minimizing the energy inside each topological sector, the solutions of (1.11) we obtain in Theorem 4 will not necessarily be local minimizers of $E_{\varepsilon}$, and are probably unstable.

\subsection{Mountain-pass solutions and threshold energies.}

We start by the crucial, although elementary, remark that when $\Lambda=\infty$, we have that $E_{\varepsilon}^{\infty}=H^{1}(\Omega)$, i.e. the whole affine space $H^{1}\left(\Omega, \mathbb{R}^{2}\right)$. This space has obviously an unique component and furthermore, given any two elements $u_{0}, u_{1} \in H^{1}\left(\Omega, \mathbb{R}^{2}\right)$ there is a natural path between them: the straight line segment $\gamma:[0,1] \longrightarrow H^{1}\left(\Omega, \mathbb{R}^{2}\right)$, defined by

$$
\gamma(s):=(1-s) u_{0}+s u_{1}, \quad \text { for } s \in[0,1] .
$$


Likewise, $F_{\varepsilon}^{\infty}=H_{g i}$, which is the projection (continuous image) of $H^{1} \times H^{1}$, and thus is connected. Given two states $\left[u_{0}\right],\left[u_{1}\right] \in H_{g i}$ we may consider the straight line between two of their representatives, $u_{0}, u_{1} \in H^{1}\left(\Omega, \mathbb{R}^{2}\right) \times H^{1}\left(\mathbb{R}^{2}, \mathbb{R}^{2}\right)$ and consider the projection in $H_{g i}$ of the straight line in $H^{1} \times H^{1}$ between $u_{0}$ and $u_{1}$.

An important example of a map of degree $n \in \mathbb{Z}$, in $H^{1}\left(\Omega, S^{1}\right) \subset$ $H^{1}\left(\Omega, \mathbb{R}^{2}\right)$ (and for which we can thus use the classical definition of the degree), is the map

$$
w_{n}(r, \theta):=\exp (\operatorname{sn} \theta)=\frac{z^{n}}{|z|^{n}} .
$$

Using (1.9) it is easy to check that $\operatorname{deg}\left(w_{n}, \Omega\right)=n$ and moreover, we can see that the energy, $E_{\varepsilon}\left(w_{n}\right)$, of the maps $w_{n}, n \in \mathbb{Z}$, is independent of $\varepsilon$ and is given by

$$
E_{\varepsilon}\left(w_{n}\right)=\frac{1}{2} \int_{\Omega}\left|\nabla w_{n}\right|^{2}=\frac{1}{2} \int_{1 / 4}^{1} r \int_{0}^{2 \pi} \frac{n^{2}}{r^{2}} d \theta d r=\pi n^{2} \log 4
$$

Hence, given $\Lambda \in \mathbb{R}^{+}$, let

$$
n_{0}:=\left[\sqrt{\frac{\Lambda}{\pi \log 4}}\right]
$$

be the largest integer less than or equal to $\sqrt{\Lambda /(6 \pi \log 4)}$. From equation (1.15) it follows that, at least for $n \in\left[-n_{0}, \ldots, n_{0}\right]$, the topological sector $\operatorname{top}_{n}\left(E_{\varepsilon}^{\Lambda}\right)$ will be non-empty, and this independently of the value of $\varepsilon>0$.

Likewise, for $F_{\varepsilon}$ we could take $w_{n}(r, \theta):=[(\exp (\imath n \theta), 0)]$. All the rest of the discussion also easily extends to the case of $F_{\varepsilon}$.

Let $\Lambda \in \mathbb{R}^{+}$be given, and let $\varepsilon<\varepsilon_{0}$ (where $\varepsilon_{0}$ is as in Theorem $1)$. Suppose that for some $n \in \mathbb{Z}$ both $\operatorname{top}_{n}\left(E_{\varepsilon}^{\Lambda}\right)$ and $\operatorname{top}_{n+1}\left(E_{\varepsilon}^{\Lambda}\right)$ are non-empty, and consider two maps

$$
u_{0} \in \operatorname{top}_{n}\left(E_{\varepsilon}^{\Lambda}\right), \quad u_{1} \in \operatorname{top}_{n+1}\left(E_{\varepsilon}^{\Lambda}\right) .
$$

Let $\gamma:[0,1] \longrightarrow H^{1}(\Omega)$ be a path between $u_{0}$ and $u_{1}$ (i.e. $\gamma(0)=u_{0}$ and $\left.\gamma(1)=u_{1}\right)$. Recall that, as we mentioned above, such a path always exists because $H^{1}\left(\Omega, \mathbb{R}^{2}\right)$ is an affine space. Then, $\gamma$ cannot be entirely contained in $E_{\varepsilon}^{\Lambda}$ - if this were so, $u_{0}$ and $u_{1}$ would be in the same path component of $E_{\varepsilon}^{\Lambda}$, and hence also in the same component of $E_{\varepsilon}^{\Lambda}$ 
which contradicts our assumption (since, by Theorem 1, the topological sectors $\operatorname{top}_{n}\left(E_{\varepsilon}^{\Lambda}\right)$ and $\operatorname{top}_{n+1}\left(E_{\varepsilon}^{\Lambda}\right)$ are disjoint open and closed subsets of the energy level set $\left.E_{\varepsilon}^{\Lambda}\right)$. Hence, there exists some $s \in(0,1)$ such that $\gamma(s) \notin E_{\varepsilon}^{\Lambda}$, which is equivalent to saying that $E_{\varepsilon}(\gamma(s)) \geq \Lambda$. A standard Min-Max argument will then yield the existence of generalized critical values of $E_{\varepsilon}$ of the form

$$
c_{n}:=\inf _{\gamma \in \mathcal{V}} \max _{s \in[0,1]} E_{\varepsilon}(\gamma(s))
$$

where $\mathcal{V}:=\left\{\gamma \in C^{0}\left([0,1], H^{1}\left(\Omega, \mathbb{R}^{2}\right)\right): \gamma(0)=u_{0}\right.$, and $\left.\gamma(1)=u_{1}\right\}$, is the space of continuous paths in $H^{1}(\Omega)$ between $u_{0}$ and $u_{1}$. The value $c_{n}$ will be a generalized critical value of $E_{\varepsilon}$. To make sure it is actually a critical value we use the following

Theorem 3. The functionals $E_{\varepsilon}$ and $F_{\varepsilon}$ satisfy the Palais-Smale condition (in $H^{1}\left(\Omega, \mathbb{R}^{2}\right)$ and $H_{g i}$, respectively).

This implies that $c_{n}$ is a critical value of $E_{\varepsilon}$ and hence, there exists a map $u \in H^{1}(\Omega)$ such that $u$ is a critical point of $E_{\varepsilon}$ and $E_{\varepsilon}(u)=c_{n}$. This $u$ is probably not a local minimum of $E_{\varepsilon}$. All this discussion extends to the case of $F_{\varepsilon}$. Thus, we have proved

Theorem 4. Suppose that for some $\Lambda \in \mathbb{R}^{+}$, we have that for some $\varepsilon<\varepsilon_{0}$ (where $\varepsilon_{0}$ is given Theorem 1) there exists $n \in \mathbb{Z}$ such that the topological sectors $\operatorname{top}_{n}\left(E_{\varepsilon}^{\Lambda}\right)$ and $\operatorname{top}_{n+1}\left(E_{\varepsilon}^{\Lambda}\right)$ are both non-empty. Then, there are mountain-pass type critical points of $E_{\varepsilon}$ or, equivalently, there exist mountain-pass type solutions of the Ginzburg-Landau equations (1.11).

More precisely, consider two maps

$$
u_{0} \in \operatorname{top}_{n}\left(E_{\varepsilon}^{\Lambda}\right) \quad \text { and } \quad u_{1} \in \operatorname{top}_{n+1}\left(E_{\varepsilon}^{\Lambda}\right),
$$

and let $c_{n}$ be defined as in (1.16). Then, there exists a map $u \in$ $H^{1}\left(\Omega, \mathbb{R}^{2}\right)$ such that $u$ is a critical point of $E_{\varepsilon}$ and $E_{\varepsilon}(u)=c_{n}$.

Likewise, if we consider two states $\Phi_{0} \in \operatorname{top}_{n}\left(F_{\varepsilon}^{\Lambda}\right)$ and $\Phi_{1} \in$ $\operatorname{top}_{n+1}\left(F_{\varepsilon}^{\Lambda}\right)$, and let $c_{n}$ be defined by

$$
c_{n}:=\inf _{\gamma \in \mathcal{V}} \max _{s \in[0,1]} F_{\varepsilon}(\gamma(s)),
$$

where now $\mathcal{V}:=\left\{\gamma \in C^{0}\left([0,1], H_{g i}\right): \gamma(0)=\Phi_{0}\right.$, and $\left.\gamma(1)=\Phi_{1}\right\}$, is the space of continuous paths in $H_{g i}$ between $\Phi_{0}$ and $\Phi_{1}$. Then, there 
exists a state $\Phi=[(u, A)] \in H_{g i}$ such that $\Phi$ is a critical point of $F_{\varepsilon}$ and $F_{\varepsilon}(\Phi)=c_{n}$.

REMARK 1. The number $c_{n}$ defined by (1.16) is called the threshold energy for the transition from the state $u_{0}$ to the state $u_{1}$. It will be the infimum of the energies for which such a transition is possible. This concept will play a crucial role in the physical behavior of our system. We will come back to this point in the remarks on physics (see below).

REMARK 2. In Theorem 4, for simplicity, we just considered transitions from a state $u_{0} \in \operatorname{top}_{n}\left(E_{\varepsilon}^{\Lambda}\right)$ to a state $u_{1}$ belonging to the adjacent state $\operatorname{top}_{n+1}\left(E_{\varepsilon}^{\Lambda}\right)$. However, both the concept of threshold energy and the result stated in Theorem 4 are immediately generalizable to the case where $u_{0} \in \operatorname{top}_{n}\left(E_{\varepsilon}^{\Lambda}\right)$ and $u_{1} \in \operatorname{top}_{k}\left(E_{\varepsilon}^{\Lambda}\right)$, for any two distinct integers $n, k \in \mathbb{Z}$. As usual, this remark and the previous one extend to the setting of the gauge-covariant functional $F_{\varepsilon}$.

REMARK 3. All these results extend to the setting of more general domains considered in Theorem 6 , stated below.

\subsection{Remarks on physics.}

\subsubsection{Ginzburg-Landau theory.}

In the Ginzburg-Landau theory of superconductivity, the conducting electrons are described as a fluid existing in two phases, the superconducting one and the normal one. In the superconducting state the material has an infinite electrical conductivity and magnetic fields are repelled from the interior of the sample (this is the so called Meissner effect).

On a microscopic scale, the superconducting state is described by the theory of Bardeen, Cooper and Schrieffer (BCS). In this theory, the existence of superconductivity is due to a pairing of the conducting electrons forming the so called Cooper pairs. For small applied forces, these pairs behave as a single particle (a boson) of twice the charge of the electron. At a macroscopic scale the behavior of the Cooper pairs is described by a complex-valued function $u$, called the condensate wave function (or order parameter). The density $|u(x)|^{2}$ is proportional to the density of pairs of superconducting electrons. 
The Ginzburg-Landau model is a phenomenological model which extends Landau's theory of second order phase transitions. It was proposed well before the microscopic theory (BCS) existed, but it can be obtained as an approximation to the macroscopic consequences of this theory. This model gives us a system of equations which describe the interaction between the condensate wave function, $u$, and the electromagnetic vector potential, $A$. In this model the parameter $\kappa=\varepsilon^{-1}$ (which depends on the material we consider and on the temperature) plays a crucial role in determining the behavior of our system.

If $\kappa<1 / \sqrt{2}$, the material is called a type I superconductor. If one applies an exterior magnetic field to the sample, then there is a critical value, $H_{c}$, such that when the applied magnetic field $H$ increases beyond $H_{c}$, the sample passes suddenly from the superconducting phase to the normal phase. On the other hand, if $\kappa \geq 1 / \sqrt{2}$, the behavior is quite different and the transition between the superconducting and the normal phase is done gradually. These materials are called type II superconductors and they are characterized by two critical values of the applied magnetic field: the first, $H_{c 1}$, corresponds to the critical field above which the two phases coexist, and the second, $H_{c 2}$, corresponds to the critical field above which all the sample will be in the normal phase. Between these two critical values the normal and superconducting phase will coexist: the normal state will be confined in vortices or filaments whose number will increase as the applied field increases. The flux lines of the magnetic field inside the material will be concentrated inside these vortices (since they are repelled by the part of the sample that is in the superconducting phase). For a detailed description of the physics involved in the phenomena of superconductivity and superfluidity see, for instance, the works of D. Saint-James, G. Sarma and E. J. Thomas [26], and of D. Tilley and T. Tilley [27]. For a more mathematical approach see the work of A. Jaffe and C. Taubes [20].

\subsubsection{Permanent currents.}

A very interesting phenomenon in superconductivity, that motivates our work, is the existence of permanent currents in a superconducting ring. The experiment is the following: a ring of superconducting material in the normal state is submitted to a fixed external magnetic field (subcritical), and then the temperature of the system is decreased until temperatures below the critical temperature corre- 
sponding to the applied field are attained. The applied field is then turned off and there is a current that persists inside the superconducting ring. Furthermore it was observed that such a current does not dissipate with time - there were experiments where the current persisted for several years without any dissipation, thus the name permanent current.

This behavior of the system indicates that we should be in presence of an energy functional having multiple wells (local minima) separated by very high barriers. The main purpose of our work is to show that even in the simple models considered in this paper, the energy functionals $E_{\varepsilon}$ and $F_{\varepsilon}$ have this type of structure.

The big height of the barriers would be associated to the "permanent" character of these currents. In fact, considering the possibility of the system tunneling through the barrier, thus moving from one energy well into another (and eventually to the ground state), the associated probability should be proportional to $\exp (-h)$, where $h$ is the height of the barrier relative to the initial state of the system. Thus, having very high barriers will yield transition probabilities close to zero and therefore justify the "permanent" character of our currents.

\subsubsection{Transitions between states and threshold energies.}

The natural question is then to describe the transitions between two different sectors - thus, the notion of threshold energy for such transitions (defined in equation (1.16)) is a crucial one for the physical behavior of our system. We remark that in the setting of the gaugeinvariant model, as we mentioned before, physical states of the system are represented by gauge-equivalence classes (defined by (1.4)) of configurations of our system - thus the configuration $(u, A)$ is just a particular representative of the state $[u, A]$. Therefore, we shouldn't consider paths between configurations in the space $H^{1}\left(\Omega, \mathbb{R}^{2}\right) \times H^{1}\left(\mathbb{R}^{2}, \mathbb{R}^{2}\right)$, but paths between states in the quotient space of $H^{1}\left(\Omega, \mathbb{R}^{2}\right) \times H^{1}\left(\mathbb{R}^{2}, \mathbb{R}^{2}\right)$ by the gauge-equivalence relation, which we denote by $H_{g i}$ (this is the physical space).

The threshold energy $c_{n}$ for a transition between a state $\left[u_{0}, A_{0}\right] \in$ $\operatorname{top}_{n}\left(F_{\varepsilon}^{\Lambda}\right)$ and a state $\left[u_{1}, A_{1}\right] \in \operatorname{top}_{n+1}\left(F_{\varepsilon}^{\Lambda}\right)$ will be of the order of $|\log \varepsilon|$. It is easy to see that it is at most of this order. Indeed, we can prove the following upper bound for the transition energy. 
Theorem 5. Let $c_{n}$ be the threshold energy for the transition between the state $\left[u_{0}, A_{0}\right] \in \operatorname{top}_{n}\left(F_{\varepsilon}^{\Lambda}\right)$ and the state $\left[u_{1}, A_{1}\right] \in \operatorname{top}_{n+1}\left(F_{\varepsilon}^{\Lambda}\right)$, defined as in (1.16). Then,

$$
c_{n} \leq M_{n}|\log \varepsilon|+L_{n},
$$

where $M_{n}$ and $L_{n}$ are constants that depend only on $n$ and our domain $\Omega$.

We will give an intuitive proof of Theorem 5 . Let $\Lambda>\pi \log (4)(n+$ $1)^{2}$ and suppose that we want to describe a path from the configuration $\left(u_{n}, A_{n}\right)=(\exp (\imath n \theta), 0) \in \operatorname{top}_{n}\left(F_{\varepsilon}^{\Lambda}\right)$ to the configuration $\left(u_{n+1}, A_{n+1}\right)=(\exp (\imath(n+1) \theta), 0) \in \operatorname{top}_{n+1}\left(F_{\varepsilon}^{\Lambda}\right)$. We remark that once we construct a path in the space $H^{1}\left(\Omega, \mathbb{R}^{2}\right) \times H^{1}\left(\mathbb{R}^{2}, \mathbb{R}^{2}\right)$ between $\left(u_{n}, A_{n}\right)$ and $\left(u_{n+1}, A_{n+1}\right)$, we can obtain a path between the corresponding physical states $\left[u_{n}, A_{n}\right]$ and $\left[u_{n+1}, A_{n+1}\right]$ in the quotient space $H_{g i}$ by projecting the original path. The general case of a transition between $\left(v_{0}, B_{0}\right) \in \operatorname{top}_{n}\left(F_{\varepsilon}^{\Lambda}\right)$ and $\left(v_{1}, B_{1}\right) \in \operatorname{top}_{n+1}\left(F_{\varepsilon}^{\Lambda}\right)$ can be proved in a similar way.

Physically, the path we construct corresponds to bringing a positive unit charge of size $\varepsilon$ from a point $P$ arbitrarily close to infinity, to the origin. By a positive unit charge of size $\varepsilon$ at a point $z_{s} \in \mathbb{C}$, we mean the map

$$
f_{z_{s}}(z)=\frac{z-z_{s}}{\left|z-z_{s}\right|} \varphi_{\varepsilon}\left(z-z_{s}\right)
$$

where $\varphi_{\varepsilon}(\cdot)=\varphi(\cdot / \varepsilon)$, and $\varphi \in C_{0}^{\infty}\left(\mathbb{R}^{2}\right)$ is such that

$$
\begin{cases}\varphi(x)=0, & \text { if }|x|<1 \\ \varphi(x)=1, & \text { if }|x|>2, \\ 0 \leq \varphi(x) \leq 1, & \text { for all } x \\ |\nabla \varphi(x)| \leq 2, & \text { for all } x\end{cases}
$$

Hence $f_{z_{s}}$ is a unit vortex at $z_{s}$ which is "smoothened out" in a ball of radius $2 \varepsilon$ around $z_{s}$. Then,

$$
F_{\varepsilon}\left(f_{z_{s}}, 0\right) \leq C_{1}|\log \varepsilon|+C_{2},
$$

where $C_{1}$ and $C_{2}$ are constants. 
Let $M \in \mathbb{R}^{+}$be an arbitrarily big number, and let $z_{s}=(1-$ $s)(-M) \in \mathbb{C}$, for $s \in[0,1]$. This will be a path from the point $(-M)$ in the negative real axis, to the origin. Using $z_{s}$ we construct the path in $H^{1}\left(\Omega, \mathbb{R}^{2}\right) \times H^{1}\left(\mathbb{R}^{2}, \mathbb{R}^{2}\right)$ defined by

$$
\left(v_{s}, B_{s}\right):=\left(f_{z_{s}} u_{n}, 0\right), \quad \text { for } s \in[0,1]
$$

We can check that $\left(v_{0}, B_{0}\right)$ is arbitrarily close in $H^{1}(\Omega)$ norm to $\left(u_{n}, A_{n}\right)$ - in fact, we would obtain the configuration $\left(u_{n}, A_{n}\right)$ if we chose $M=$ $+\infty$. Hence, in particular, for big values of $M$, we certainly have $\left(v_{0}, B_{0}\right) \in \operatorname{top}_{n}\left(F_{\varepsilon}^{\Lambda}\right)$. Furthermore, $\left(v_{1}, B_{1}\right)=\left(u_{n+1}, A_{n+1}\right)$ and we can obtain estimate (1.18) as a consequence of the bound (1.21).

Hence we see that the path corresponding to passing a positive unit charge "of size $\varepsilon$ " from the outside of our annulus, to the hole inside the annulus, corresponds to increasing by one the degree of our map and requires that we go to an energy level of order $|\log \varepsilon|$. To prove that any transition between $\operatorname{top}_{n}\left(F_{\varepsilon}^{\Lambda}\right)$ and $\operatorname{top}_{n+1}\left(F_{\varepsilon}^{\Lambda}\right)$ also requires passing through energy levels of order $|\log \varepsilon|$, thus proving that $c_{n}$ is of order $|\log \varepsilon|$, is a very delicate problem. We will show a way to solve this problem and obtain very precise estimates for the threshold energies in a forthcoming work ([1]).

\subsection{The case of more general domains.}

In Theorem 1 we considered a very particular domain - the annulus $\Omega=\left\{x \in \mathbb{R}^{2}: 1 / 4<|x|<1\right\}$. However, once we have the result for the annulus, it is not hard to extend it to the case of a general open subset $D \subset \mathbb{R}^{2}$, or even the case of a domain in a Riemannian manifold $\mathcal{M}$. We define the energy functional just as in (1.1) but replacing $\Omega$ by our new domain $D$,

$$
E_{\varepsilon}(u, D)=\frac{1}{2} \int_{D}|\nabla u|^{2}+\frac{1}{4 \varepsilon^{2}} \int_{D}\left(1-|u|^{2}\right)^{2},
$$

and we define the corresponding level sets

$$
E_{\varepsilon}^{\Lambda}(D):=\left\{u \in H^{1}\left(D, \mathbb{R}^{2}\right): E_{\varepsilon}(u, D)<\Lambda\right\} .
$$

We start by fixing a set of representatives of generators of $\pi_{1}(D)$ (the first homotopy group of $D),\left\{\gamma_{j}, j \in J\right\}$, such that each $\gamma_{j}: S^{1} \longrightarrow D$, 
is an injective closed smooth curve inside our open set $D$. Hence, $\gamma_{j}$ will have a tubular neighborhood $\Gamma_{j} \subset \Omega$. We may suppose that for each $j$ there is a positive number, $\delta_{j}>0$, such that for each $j$

i) $\Gamma_{j}=\left\{x \in D: \operatorname{dist}\left(x, \gamma_{j}\right)<\delta_{j}\right\}$.

ii) There is a diffeomorphism

$$
\Phi_{j}: \Gamma_{j} \longrightarrow S^{1} \times(0,1),
$$

such that $\gamma_{j}(\theta)=\Phi_{j}^{-1}(\theta, 1 / 2)$, and the Jacobian of $\Phi_{j}$ is uniformly bounded from above and away from zero, i.e. there is a constant $C_{j}>0$ such that

$$
\frac{1}{C_{j}}<\left|\nabla \Phi_{j}(x)\right|<C_{j}, \quad \text { for all } x \in \Gamma_{j} .
$$

Let $\hat{\Omega}:=S^{1} \times(1 / 4,3 / 4)$. This set is topologically an annulus just like our standard set $\Omega$ considered before. Let $Y_{j}:=\Phi_{j}^{-1}(\hat{\Omega})$. Given a map $u \in E_{\varepsilon}^{\Lambda}(D)$ we consider the map $w_{j}=u \circ \Phi_{j}^{-1}: \hat{\Omega} \longrightarrow \mathbb{R}^{2}$. The map $w_{j}$ belongs to $E_{\varepsilon}(\hat{\Omega})$, where $\varrho$ is a constant that depends only on $\Lambda$ and the constant $C_{j}$ in (1.24). Thus, we can apply Theorem 1 replacing $\Omega$ and $\Lambda$ by $\hat{\Omega}$ and $\varrho$, respectively. Hence for $\varepsilon$ sufficiently small $\operatorname{deg}\left(w_{j}, \hat{\Omega}\right)$ is well defined. We set, for each $j \in J$,

$$
\operatorname{deg}\left(u, Y_{j}\right):=\operatorname{deg}\left(w_{j}, \hat{\Omega}\right) .
$$

Suppose that the index set $J$ is finite $(J=\{1, \ldots, m\})$, i.e. suppose that we fix a finite number of (representatives of) generators of $\pi_{1}(D)$. We define the topological type of $u \in E_{\varepsilon}^{\Lambda}(D)$ as the $m$-tuple of integers

$$
\chi(u):=\left(\operatorname{deg}\left(u, Y_{1}\right), \ldots, \operatorname{deg}\left(u, Y_{m}\right)\right) .
$$

By the previous argument, this $\chi(u) \in \mathbb{Z}^{m}$ is well defined for sufficiently small $\varepsilon$. The continuity of $\chi$ in $W^{1,2}\left(D, \mathbb{R}^{2}\right)$ topology inside $E_{\varepsilon}^{\Lambda}(D)$ (which is an immediate consequence of the continuity of $\operatorname{deg}(u, \Omega$ ) proved in section 7 ) will then allow us to assert that, since $\mathbb{Z}^{m}$ is discrete, for each $P \in \mathbb{Z}^{m}$, its inverse image by $\chi$, i.e. $\chi^{-1}(P)=\{u \in$ $\left.E_{\varepsilon}^{\Lambda}(D): \chi(u)=P\right\}$, will be an open and closed subset of $E_{\varepsilon}^{\Lambda}(D)$. For each $P \in \mathbb{Z}^{m}$, we call $\chi^{-1}(P)$ the $P$-topological sector of $E_{\varepsilon}^{\Lambda}(D)$. We have thus proved the following Theorem which extends the classification given by Theorem 1 to this more general setting. 
Theorem 6. Let $D$ be an open subset of $\mathbb{R}^{2}$ or a domain in a Riemann manifold $\mathcal{M}$. Let $\gamma_{1}, \ldots, \gamma_{m}$ be simple, closed and smooth curves which are a set of representatives of generators of $\pi_{1}(D)$. Given $\Lambda>0$ there exists $\varepsilon_{0}>0$, depending on $\Lambda$, such that for $\varepsilon<\varepsilon_{0}$ we can define a continuous map

$$
\begin{aligned}
& \chi: E_{\varepsilon}^{\Lambda}(D) \longrightarrow \mathbb{Z}^{m}, \\
& u \longmapsto\left(\operatorname{deg}\left(u, Y_{1}\right), \ldots, \operatorname{deg}\left(u, Y_{m}\right)\right),
\end{aligned}
$$

such that for the special case where $u \in E_{\varepsilon}^{\Lambda}(D) \cap W^{1,2}\left(D, S^{1}\right)$, we recover the classical notion of degree of a $S^{1}$ valued map. Therefore, given $P=\left(P_{1}, \ldots, P_{m}\right) \in \mathbb{Z}^{m}$, the subset $\chi^{-1}(P) \subset E_{\varepsilon}^{\Lambda}(D)$ will be an open and closed subset of $E_{\varepsilon}^{\Lambda}(D)$.

The same argument in the context of the superconductivity model will give a similar extension of Theorem 2 .

\subsection{Idea of the proof of Theorem 1 .}

The maps $u \in E_{\varepsilon}^{\Lambda}$ may take values close to zero, which creates big technical problems for defining their degree. However, this can only happen in a set of small measure. We will start by studying, in sections 2,3 and 4 the set $G(\zeta)$ where $|u|$ is smaller than an appropriately chosen $\zeta \in(1 / 2,3 / 4)$. For technical reasons (to avoid problems that may appear near the boundary $\partial \Omega$ ) we will concentrate on the components of $G(\zeta)$ that intersect an interior annulus

$$
Y:=\left\{x \in \mathbb{R}^{2}: \frac{1}{2}<|x|<\frac{3}{4}\right\}
$$

Using Sard's Lemma we will see that for sufficiently small $\varepsilon$, these components of $G$ may be included in a finite number of simply-connected sets, which we denote by $W_{k}, k=1, \ldots, \tilde{N}$. Their boundaries will be closed smooth curves, $V_{k}=\partial W_{k}$, and $|u|=\zeta$ on each of the $V_{k}$ 's.

In Section 2 we see, using the coarea formula, that the sum of the lengths of the $V_{k}$ 's will tend to zero when $\varepsilon \longrightarrow 0$. Furthermore, the coarea formula also gives us a bound on the $L^{1}$ norm of $\nabla u$ on $V=\bigcup V_{k}$. Since $|u|=\zeta>1 / 2$ on $V_{k}$, it makes sense to talk about $\operatorname{deg}\left(u, V_{k}\right)$. 
In Section 3 we the obtain an uniform bound on $\sum\left|\operatorname{deg}\left(u, V_{k}\right)\right|$ using the estimate for $\|\nabla u\|_{L^{1}(V)}$ (and consequently we will also have uniform bounds on $\left|\operatorname{deg}\left(u, V_{k}\right)\right|$ for each $\left.k\right)$. Thus, we see that for all $u \in E_{\varepsilon}^{\Lambda}$ the number of $V_{k}$ 's such that $\operatorname{deg}\left(u, V_{k}\right) \neq 0$ (which we call the "charged" $V_{k}$ 's) is uniformly bounded by a constant depending only on $\Lambda$. Suppose that the charged $V_{k}$ 's are $V_{1}, \ldots, V_{N_{2}}$.

In Section 4 we will focus our attention on the "uncharged" $V_{k}$ 's (i.e. those for which $\operatorname{deg}\left(u, V_{k}\right)=0$ ). We will see, again using the estimate for $\|\nabla u\|_{L^{1}(V)}$ obtained in Section 2, that the number of "uncharged" $V_{k}$ 's such that the oscillation of $u$ is bigger than or equal to $\pi / 3$, is also uniformly bounded. Suppose they are $V_{N_{2}+1}, \ldots, V_{N}$. Moreover, for the remaining $V_{k}$ 's, i.e. the "uncharged" ones such that the oscillation of $u$ is smaller than $\pi / 3$ (which will be $V_{N+1}, \ldots, V_{\tilde{N}}$ ), we are able to prove that the energy minimizing extension to $W_{k}$ of $u_{\mid V_{k}}$ will have absolute value which is uniformly bounded away from zero hence we will show that these sets are rather "harmless".

In Section 5, thanks to the uniform bound on $N$ (the number of "charged" $V_{k}$ 's plus that of "uncharged" $V_{k}$ 's such that the oscillation of $u$ is bigger than or equal to $\pi / 3$ ), we can cover $V_{1}, \ldots, V_{N}$ by a finite (uniformly bounded) number of balls, $B_{1}, \ldots, B_{m}$, of radius of order at most $\varepsilon^{\alpha}$ for some $\alpha>1 / 2$, and which are far away from each other (in the sense that suitable dilations of the $B_{i}$ 's are pairwise disjoint). Furthermore, we will see that $\operatorname{deg}\left(u, \partial B_{i}\right)=0$, for all $i$. This means that though we may have individual singularities that are charged, at a scale of order $\varepsilon^{1 / 2}$ they cluster to form neutral structures.

In Section 6 we will finally give the good definition of the global degree of $u$ in $\Omega, \operatorname{deg}(u, \Omega)$. Let

$$
T:=\left\{r \in\left(\frac{1}{2}, \frac{3}{4}\right) \text { such that } S_{r} \cap G(\zeta) \neq \varnothing\right\},
$$

and let

$$
A:=\left(\frac{1}{2}, \frac{3}{4}\right) \backslash T \text {. }
$$

We show that $|T| \longrightarrow 0$, when $\varepsilon \longrightarrow 0$, and hence $|A| \longrightarrow 1 / 4$, when $\varepsilon \longrightarrow 0$. For $r \in A$ we define

$$
f(r):=\operatorname{deg}\left(u, S_{r}\right)=\operatorname{deg}\left(\frac{u}{|u|}, S_{r}\right) \in \mathbb{Z} .
$$

This function is well defined since for $r \in A,|u(r, \theta)| \geq \zeta$. As we mentioned before, for $u \in W^{1,2}\left(\Omega, S^{1}\right)$ this function is constant. In 
our case this might not be true, but by the results of Section 5, it cannot change too much: as a matter of fact, for $\varepsilon$ sufficiently small, the value of $f$ can only change when $S_{r}$ intersects one of the balls $B_{i}$, and even when this occurs, the absolute value of $f$ remains bounded by a constant that depends only on $\Lambda$. Outside these balls (i.e. when $S_{r} \cap B=\varnothing$, where $\left.B:=\bigcup B_{i}\right) f(r)$ will always have the same value (since $\operatorname{deg}\left(u, \partial B_{i}\right)=0$ ). This is the value we use to define $\operatorname{deg}(u, \Omega$ ), which will thus automatically be an integer. To recover this integer we can also integrate $f(r)$ over $A$ and divide by the measure of $A$, thus defining

$$
\widetilde{\operatorname{adeg}}(u, \Omega):=\frac{1}{|A|} \int_{A} f(r) d r
$$

This quantity, $\widetilde{\operatorname{adeg}}(u, \Omega)$, is called the approximate degree of $u$ in $\Omega$. In general, it is not an integer, but it will tend to the integer $\operatorname{deg}(u, \Omega)$ as $\varepsilon \longrightarrow 0$. In fact, let $Q=A \cap B=\bigcup\left(A \cap B_{i}\right)$. The measure of $Q$ tends to zero when $\varepsilon \longrightarrow 0$ (it is bounded by $|B|$ which, in turn, is at most, of order $\varepsilon^{\alpha}<\varepsilon^{1 / 2}$ ). Furthermore, $f$ remains uniformly bounded even inside $Q$, and hence, we can see that

$$
\widetilde{\operatorname{adeg}}(u, \Omega)-\operatorname{deg}(u, \Omega) \mid<\frac{1}{4}
$$

for sufficiently small $\varepsilon$. Thus we can recover the integer $\operatorname{deg}(u, \Omega)$ as the closest integer to $\widetilde{\operatorname{adeg}}(u, \Omega)$ for $\varepsilon$ small.

In Section 7 we will prove, for sufficiently small $\varepsilon$, the continuity of $\widetilde{\operatorname{adeg}}(u, \Omega)$ (and thus also of $\operatorname{deg}(u, \Omega)$ ) in $W^{1,2}(\Omega)$ norm, inside the level set $E_{\varepsilon}^{\Lambda}$ we fixed. Using this continuity we will then conclude the proof of Theorem 1 in Section 5.

Finally, in the Appendix (Section 12) we prove a general covering Lemma of which we used a special case to obtain the balls $B_{i}$ in Section 5 .

\subsection{Open questions and related results.}

As we saw, many questions about this subject remain open, in particular in the borderline between the mathematics and the physical behavior of these systems, a considerable amount of work remains to be done. In this subsection we will discuss some of these problems shortly 
and mention some results of related interest. We start by mentioning a few problems we are working on at the moment.

In [1] we are able to carry out a more detailed study of the properties of the threshold energies we introduced above. In particular, using some techniques introduced by F. Bethuel and the author in [4], we can prove a more accurate version of the upper bound for the threshold energy $c_{n}$ stated in Theorem 5 . More precisely, we show that there exists a constant $\alpha_{n}$, not depending on $\varepsilon$, such that $c_{n} \leq \pi|\log \varepsilon|+\alpha_{n}$.

This estimate is crucial to succeeding in obtaining (see [1]) a lower bound for $c_{n}$ which is of the same order of the above, i.e. to showing that $c_{n} \geq \pi|\log \varepsilon|-\alpha_{n}$. Such a bound, as we mentioned, implies that the energy barriers have a height of at least $\pi|\log \varepsilon|-\alpha_{n}$, and therefore, since $\varepsilon$ is supposed to be small, we will have very high barriers separating the wells. This agrees with what we expected considering the physical behavior of our system, as we described above.

Regarding the extension of our results to the 3-dimensional case, there is a substantial part we are able to do, but there are still some technical difficulties (which stem from the higher degree of liberty of the equivalent of the $V_{k}$ 's, which, in this setting, will be two-dimensional surfaces). Once we succeed in defining the degree, we can obtain mountain-pass solutions just as for the dimension 2, but proving that the threshold energy, $c_{n}$, is of order $|\log \varepsilon|$ should be considerably harder (for results on the structure of the singularities of the Abelian Higgs model in $\mathbb{R}^{3}$, see the works of $T$. Rivière [23] and [24]).

Our work was also motivated by the paper of S. Jimbo and Y. Morita [16]. In [16] the authors establish the existence of stable nontrivial solutions for the Ginzburg-Landau equations in the case the domain $\Omega \subset \mathbb{R}^{3}$ is a solid of revolution obtained by rotating a convex cross-section around the z-axis in $\mathbb{R}^{3}$. Thanks to this special geometry, they can find solutions using a separation of variables method. They show that the solutions constructed are stable for variations in a linear space that is transversal to the gauge-invariance of the problem.

Very recently, while this work was being finished, the author received a series of preprints of S. Jimbo, Y. Morita and J. Zhai [17], [18], [19] where they improve the techniques developed in [16] and introduce some new ideas to obtain very interesting results about stationary solutions of the Ginzburg-Landau equations in topologically non-trivial domains. The author also received recently a preprint J. Rubinstein and P. Sternberg [25], where the ideas of B. White and F. Bethuel concerning the homotopy classes for Sobolev functions are used, together 
with variational techniques, in a very ingenious way, to obtain a homotopy classification for the minimizers of the Ginzburg-Landau energy in the case the domain is topologically a torus in $\mathbb{R}^{3}$. One fundamental difference between these works and ours is that, since their authors are looking at critical points, they rely strongly on the Ginzburg-Landau equation to prove nice properties for these critical points, and then succeed in defining the degree of the stationary solutions using these properties. In our case, since we look at the whole level set of the energy, we cannot rely on the equation to help us define the degree. This, as we saw, poses many technical problems, but gives us a considerable amount of new information. Such information should enable us to have a better understanding about the formation of permanent currents and the transition processes between physical states.

Another important question is that of the evolution equation for Ginzburg-Landau. Recently there was some work of F. H. Lin [21], [22], and of S. Demoulini and D. Stuart [12] on the heat flow for GinzburgLandau. The author, F. Bethuel and Y. Guo have also obtained some results regarding the dynamical stability of symmetric vortices in the Maxwell-Higgs model (see [15] and [5]).

\section{REMARKS ON NOTATION.}

- $\Omega$ is the annulus $\left\{x \in \mathbb{R}^{2}: 1 / 4<|x|<1\right\} \subset \mathbb{R}^{2}$. Its boundary, $\partial \Omega$, has two connected components: $\partial \Omega_{1}=S_{1 / 4}$, the inner circle, and $\partial \Omega_{2}=S_{1}$, the exterior circle. On $\partial \Omega, \nu(x)$ stands for the exterior unit normal to $\partial \Omega$ at $x$. Hence $\nu(x)=-x /|x|$ on $\partial \Omega_{1}$, and $\nu(x)=x /|x|$ on $\partial \Omega_{2}$. For $x \in \partial \Omega, \tau(x)$ stands for the unit tangent vector to $\partial \Omega$ at $x$, pointing in the sense of increasing $\theta$.

- $\wedge$ denotes the wedge product of differential forms, and $\times$ represents the exterior product of two vectors in $\mathbb{R}^{2}$ (it is considered as a real number).

- We often use the natural identification between an one-form and the associated vector (given by the scalar product in $\mathbb{R}^{2}$ ).

- Although we would normally prefer to write vectors as columns, we will often write them as rows because it makes it easier to insert them in the text.

- We identify the vector $\left(v^{1}, v^{2}\right) \in \mathbb{R}^{2}$ with the complex number $v^{1}+\imath v^{2}$. The scalar product in $\mathbb{C}$ is denoted by $($,$) . So (u, v)=$ $(u \bar{v}+v \bar{u}) / 2$. With this notation we have that $u \times u_{\tau}=\left(\imath u, u_{\tau}\right)$. 
Although this permanent switch between the vector and the complex number notation may be slightly confusing at the beginning, later on the reader will appreciate the convenience that stems from having both notations available.

- $d$ denotes the exterior derivative and $\star$ denotes the Hodge star operator, which in $\mathbb{R}^{2}$ is the linear operator on $\mathbb{R}$-valued forms defined by

$$
\star 1=d x^{1} \wedge d x^{2}, \star d x^{1}=d x^{2}, \star d x^{2}=d x^{1}, \text { and } \star d x^{1} \wedge d x^{2}=1 .
$$

We have that for $k$-forms on $\mathbb{R}^{2}, \star \star=I^{(k(2-k))}$, where $I$ denotes the identity. Hence $\star \star \alpha=\alpha$, if $\alpha$ is a zero-form or a two-form, and $\star \star \alpha=-\alpha$, if $\alpha$ is a one-form.

- $d^{\star}$ denotes the operator $\star^{-1} d \star$, where $\star^{-1}$ stands for the inverse operator of $\star$.

- In many of the estimates we obtain during the proof of Theorem 1 , there are constants which depend on the domain considered. However, since we will have fixed as domain the annulus $\Omega$, we will usually not mention such dependence explicitly in the text.

\section{Coarea formula and control of the bad set.}

As we mentioned before, the bad set consists of the places where $|u|$ is close to zero. Nevertheless, the presence of the potential term in $E_{\varepsilon}$ (in particular, the presence of the $\varepsilon^{-2}$ factor), assures us that for $u \in E_{\varepsilon}^{\Lambda}$, the measure of the set $\{x:|u|<1 / 2\}$ will be very small when $\varepsilon \longrightarrow 0$. In fact, as we will see in this section, a more careful analysis using the coarea formula will allow us to prove much more about this set.

Suppose $\Lambda$ and $\varepsilon$ given and fix an element $u \in E_{\varepsilon}^{\Lambda} \cap C^{\infty}(\Omega)$. For each $\zeta \in[1 / 2,3 / 4]$, let

$$
V(\zeta)=\{x \in \Omega:|u(x)|=\zeta\} .
$$

By Sard's Lemma we know that for almost every $\zeta, V(\zeta)$ is a onedimensional submanifold of $\Omega$, hence we will suppose that the $\zeta$ we choose is in these conditions. We will now define as our bad set, the set $G$ where $|u|$ is smaller than $\zeta$. Let

$$
G(\zeta):=\{x \in \Omega:|u(x)|<\zeta\}, \quad \zeta \in\left[\frac{1}{2}, \frac{3}{4}\right] .
$$


It is easy to see that for small $\varepsilon$, the measure of $G(\zeta)$ will be very small. In fact,

$$
\begin{aligned}
\int_{G(\zeta)}\left(1-|u|^{2}\right)^{2} & \geq \int_{G(\zeta)}\left(1-\zeta^{2}\right)^{2} \\
& \geq|G(\zeta)|\left(1-\zeta^{2}\right)^{2} \\
& \geq\left(\frac{7}{16}\right)^{2}|G(\zeta)|
\end{aligned}
$$

and,

$$
\int_{G(\zeta)}\left(1-|u|^{2}\right)^{2} \leq 4 \varepsilon^{2} \frac{1}{4 \varepsilon^{2}} \int_{\Omega}\left(1-|u|^{2}\right)^{2} \leq 4 \varepsilon^{2} \Lambda
$$

Combining (2.1) and (2.2) we obtain the desired bound on $|G(\zeta)|$,

$$
|G(\zeta)| \leq\left(\frac{16}{7}\right)^{2} 4 \varepsilon^{2} \Lambda=C \varepsilon^{2} \underset{\varepsilon \rightarrow 0}{\longrightarrow} 0,
$$

where $C$ is a constant depending only on the energy bound $\Lambda$.

\subsection{The coarea formula.}

Using the coarea formula of Federer and Flemming, we can obtain a considerable amount of information about the $V_{k}$ 's and the behavior of $u_{\mid V_{k}}$, for $\zeta$ conveniently chosen.

Here we will apply a special case of this formula which can be stated as follows (for a proof and more general forms of this result see, for instance, L. Evans and R. Gariepy [13]).

Theorem 7 (coarea formula (change of variables)). Let $f: \mathbb{R}^{2} \longrightarrow \mathbb{R}$ be Lipschitz. Then, for every Lebesgue summable function $g: \mathbb{R}^{2} \longrightarrow \mathbb{R}$, we have that

i) The restriction $g_{\mid f^{-1}\{y\}}$ is Hausdorff $\mathcal{H}^{1}$-measurable for almost every $y$.

ii) For every measurable set $X \subset \mathbb{R}^{2}$,

$$
\int_{X} g|\nabla f| d x=\int_{\mathbb{R}}\left(\int_{f^{-1}\{y\} \cap X} g d \mathcal{H}^{1}\right) d y .
$$


REMARK. By Rademacher's Theorem, since $f$ is Lipschitz, it is differentiable almost everywhere, and hence $\nabla f$ is defined almost everywhere $x \in X$.

\subsection{Upper-bound for the length of the $V_{k}$ 's.}

We start by proving that the length (Hausdorff one-dimensional measure) of the $V_{k}$ 's is small for small $\varepsilon$. As a matter of fact, if we denote $\Xi:=\{x: 1 / 2 \leq|u| \leq 3 / 4\}$, it follows from the co-area formula that

$$
\begin{aligned}
\int_{1 / 2}^{3 / 4} \mathcal{H}^{1}(V(\zeta)) d \zeta & =\int_{\Xi}|\nabla| u|| \\
& \leq \int_{\Xi}|\nabla u| \\
& \leq\left(\int_{\Xi}|\nabla u|^{2}\right)^{1 / 2}|\Xi|^{1 / 2}
\end{aligned}
$$

where we used Cauchy-Schwarz for the last inequality. Moreover,

$$
\frac{1}{2} \int_{\Omega}|\nabla u|^{2} \leq E_{\varepsilon}(u) \leq \Lambda,
$$

hence,

$$
\left(\int_{\Omega}|\nabla u|^{2}\right)^{1 / 2} \leq \sqrt{2 \Lambda}
$$

On the other hand, the measure of $\Xi$ can also be estimated using the energy bound (just like we did for $G(\zeta)$, in fact $\Xi=G(1 / 2)$ ). We obtain

$$
|\Xi| \leq\left(\frac{16}{7}\right)^{2} \int_{\Xi}\left(1-|u|^{2}\right)^{2} \leq\left(\frac{32}{7}\right)^{2} \varepsilon^{2} \Lambda
$$

From (2.4), (2.5) and (2.6), it follows that

$$
\int_{1 / 2}^{3 / 4} \mathcal{H}^{1}(V(\zeta)) d \zeta \leq \frac{32 \sqrt{2}}{7} \varepsilon \Lambda .
$$


Hence, except for $\zeta$ in a set $Z_{1} \subset[1 / 2,3 / 4]$ of measure at most $\sqrt{2} / 70 \leq$ $1 / 40$

$$
\mathcal{H}^{1}(V(\zeta)) \leq \frac{70}{\sqrt{2}} \frac{32 \sqrt{2}}{7} \Lambda \varepsilon=320 \Lambda \varepsilon
$$

\subsection{Upper-bound for the $L^{1}(V(\zeta))$ norm of $\nabla u$.}

A different application of the coarea formula yields

$$
\int_{0<\zeta<1} \int_{V(\zeta)}|\nabla u|=\int_{\Omega}|\nabla| u|||\nabla u| \leq \int_{\Omega}|\nabla u|^{2}
$$

Since we assume that $u \in E_{\varepsilon}^{\Lambda}$, from (2.8) it follows that

$$
\int_{0<\zeta<1} \int_{V(\zeta)}|\nabla u| \leq 2 E_{\varepsilon}^{\Lambda}(u) \leq 2 \Lambda
$$

Using Fubini's Theorem, we will then have that except for $\zeta$ in a set $Z_{2} \subset[1 / 2,3 / 4]$ of measure at most $1 / 40$,

$$
\int_{V(\zeta)}|\nabla u| \leq 80 \Lambda
$$

Thus, except when $\zeta$ belongs to the set $Z_{1} \cup Z_{2}$, whose measure is at most $1 / 20$, estimates $(2.7)$ and $(2.10)$ will be valid. For the rest of this paper we will choose a $\zeta \in(1 / 2,3 / 4)$ such that estimates (2.7) and (2.10) are valid, and that $V(\zeta)$ is a one-dimensional submanifold of $\Omega$. Hence, $V(\zeta)$ consists of a finite number of simple curves in $\Omega$. Let $V_{1}, \ldots, V_{\breve{N}}$, denote the connected components of $V(\zeta)$. Equation (2.7) gives us an upper-bound on the length of each $V_{k}$,

$$
\sum_{k=1}^{\breve{N}} \mathcal{H}^{1}\left(V_{k}\right) \leq \mathcal{H}^{1}(V(\zeta)) \leq 320 \Lambda \varepsilon
$$

In particular,

$$
\mathcal{H}^{1}\left(V_{k}\right) \leq 320 \Lambda \varepsilon, \quad \text { for all } k=1, \ldots, \breve{N} .
$$


Hence, for small $\varepsilon$, the length of each $V_{k}$ will be small (the same being true for the sum of their lengths).

\section{Properties of the $V_{k}$ 's which are far from $\partial \Omega$.}

We consider the interior subdomain $Y:=\{(r, \theta): 1 / 2<r<$ $3 / 4\} \subset \Omega$, i.e., the interior annulus consisting of the points whose distance to the origin lies between $1 / 2$ and $3 / 4$. For technical reasons, we will also have to consider a slightly enlarged subdomain, $\hat{Y}:=\{(r, \theta)$ : $3 / 8<r<7 / 8\}$. Hence, $Y \Subset \hat{Y} \Subset \Omega$.

We start by proving that for $\varepsilon$ sufficiently small, the $V_{k}$ 's that intersect $\hat{Y}$ are closed curves that stay away from the boundary of $\Omega$.

Lemma 1. If $\varepsilon$ is sufficiently small, then $V_{k} \cap \hat{Y} \neq \varnothing$, implies that $V_{k}$ is a closed curve and dist $\left(V_{k}, \partial \Omega\right)>1 / 16$.

Proof. Suppose that $V_{k} \cap \hat{Y} \neq \varnothing$. Then, since dist $(\hat{Y}, \partial \Omega)=1 / 8$, for $\operatorname{dist}\left(V_{k}, \partial \Omega\right)$ to be smaller than $1 / 16$, it is necessary that $\operatorname{diam}\left(V_{k}\right) \geq$ $1 / 16$. However, from (2.12) it follows that

$$
\operatorname{diam}\left(V_{k}\right) \leq \mathcal{H}^{1}\left(V_{k}\right) \leq 320 \Lambda \varepsilon
$$

Hence, for $\varepsilon<\Lambda / 5120$ we must have that $\operatorname{diam}\left(V_{k}\right)<1 / 16$, and thus, $\operatorname{dist}\left(V_{k}, \partial \Omega\right)>1 / 16$.

The fact that $V_{k}$ is then a closed curve, follows from it being a one-dimensional submanifold of $\Omega$ which does not touch $\partial \Omega$.

Henceforth, we will always suppose that $\varepsilon$ is chosen sufficiently small for the result in Lemma 1 to be true. Suppose that the $V_{k}$ 's that intersect $\hat{Y}$ are $V_{1}, \ldots, V_{\bar{N}}$. They will be closed curves and thus, by Jordan's Curve Theorem, we can define the domain $W_{k}$ enclosed by $V_{k}$ ( $W_{k}$ is the bounded component of $\mathbb{R}^{2} \backslash V_{k}$, and in particular, $\left.V_{k}=\partial W_{k}\right)$.

Among $V_{1}, \ldots, V_{\bar{N}}$ we will only consider those which are maximal in the following sense: for $i, j \leq \bar{N}$, if $V_{i} \subset W_{j}$ then we disregard $V_{i}$ and just keep $V_{j}$ in our list (so we always keep only the exterior curves). Suppose that $V_{1}, \ldots, V_{\tilde{N}}$, for some $\tilde{N} \leq \bar{N}$, are the maximal curves we obtain. These are the $V_{k}$ 's that will interest us for the rest of this paper (unless stated otherwise, henceforth we will always assume $k \leq \tilde{N}$ ). 


\subsection{Estimates for $\operatorname{deg}\left(u, V_{k}\right)$.}

By the definition of $V(\zeta)$, the restriction of $u$ to $V_{k}$ will have values in the circle of radius $\zeta$, i.e. $u_{\mid V_{k}}: V_{k} \longrightarrow S_{\zeta}$, where we denote $S_{\zeta}=$ $\left\{z \in \mathbb{R}^{2}:|z|=\zeta\right\}$. Therefore, we can define the degree of $u$ : as usual we consider the map

$$
v=\left(\begin{array}{c}
v^{1} \\
v^{2}
\end{array}\right):=\frac{u}{|u|}: V_{k} \longrightarrow S^{1},
$$

and we define

$$
\operatorname{deg}\left(u, V_{k}\right):=\operatorname{deg}\left(v, V_{k}\right):=\frac{1}{2 \pi} \int_{V_{k}} v \times \frac{\partial v}{\partial \tau} d \tau,
$$

where $\tau$ denotes, as usual, the arc-length parameter on $V_{k}$.

Since $u=|u| v$, we have that

$$
\nabla u=\nabla(|u| v)=\left(\begin{array}{ll}
\frac{\partial|u|}{\partial x^{1}} v^{1}+|u| \frac{\partial v^{1}}{\partial x^{1}} & \frac{\partial|u|}{\partial x^{2}} v^{1}+|u| \frac{\partial v^{1}}{\partial x^{2}} \\
\frac{\partial|u|}{\partial x^{1}} v^{2}+|u| \frac{\partial v^{2}}{\partial x^{1}} & \frac{\partial|u|}{\partial x^{2}} v^{2}+|u| \frac{\partial v^{2}}{\partial x^{2}}
\end{array}\right)
$$

Thus,

$$
\begin{aligned}
|\nabla u|^{2}= & |u|^{2}\left(\left(\frac{\partial v^{1}}{\partial x^{1}}\right)^{2}+\left(\frac{\partial v^{1}}{\partial x^{2}}\right)^{2}+\left(\frac{\partial v^{2}}{\partial x^{1}}\right)^{2}+\left(\frac{\partial v^{2}}{\partial x^{2}}\right)^{2}\right) \\
(3.3)+ & \left(\left(v^{1}\right)^{2}+\left(v^{2}\right)^{2}\right) \\
& \cdot\left(\left(\frac{\partial|u|}{\partial x^{1}}\right)^{2}+\left(\frac{\partial|u|}{\partial x^{2}}\right)^{2}\right)+|u| \frac{\partial|u|}{\partial x^{1}}\left(v \frac{\partial v}{\partial x^{1}}\right)+|u| \frac{\partial|u|}{\partial x^{2}}\left(v \frac{\partial v}{\partial x^{2}}\right) .
\end{aligned}
$$

But since $|v|=C^{t e}=1$, it follows that

$$
\left(v^{1}\right)^{2}+\left(v^{2}\right)^{2}=|v|^{2}=1,
$$

and,

$$
v \frac{\partial v}{\partial x^{i}}=\frac{1}{2} \frac{\partial}{\partial x^{i}}(v v)=0
$$

Thus, (3.3) yields

$$
|\nabla u|^{2}=|u|^{2}|\nabla v|^{2}+|\nabla| u||^{2} .
$$


Hence, in particular,

$$
|\nabla u|^{2} \geq|u|^{2}|\nabla v|^{2}
$$

For $x \in V_{k}$, since $|u(x)|=\zeta \geq 1 / 2$, this yields

$$
|\nabla u|^{2} \geq \zeta^{2}|\nabla v|^{2} \geq \frac{1}{4}|\nabla v|^{2}
$$

which, in turn, implies that on $V_{k}$,

$$
|\nabla u| \geq \frac{1}{2}|\nabla v|
$$

From equations (3.1) and (3.7) it follows that

$$
\left|\operatorname{deg}\left(u, V_{k}\right)\right|=\left|\operatorname{deg}\left(v, V_{k}\right)\right| \leq \int_{V_{k}}\left|v \times \frac{\partial v}{\partial \tau}\right| d \tau \leq \int_{V_{k}}|\nabla v| \leq 2 \int_{V_{k}}|\nabla u| .
$$

Therefore, using equation (2.10), we obtain a bound on the absolute value of the degree of $u$ in each of the $V_{k}$, for all $k=1, \ldots, \tilde{N}$ (we remark that this bound is also valid for $\tilde{N}<k \leq \hat{N}$ as long as $V_{k}$ is a closed curve - so that we have no problem defining $\operatorname{deg}\left(u, V_{k}\right)$ ),

$$
\left|\operatorname{deg}\left(u, V_{k}\right)\right| \leq 2 \int_{V_{k}}|\nabla u| \leq 2 \int_{V(\zeta)}|\nabla u| \leq 160 \Lambda
$$

Moreover, we even have a bound on the sum of the absolute values of these degrees,

$$
\sum_{k=1}^{\tilde{N}}\left|\operatorname{deg}\left(u, V_{k}\right)\right| \leq 2 \sum_{k=1}^{\tilde{N}} \int_{V_{k}}|\nabla u| \leq 2 \int_{V(\zeta)}|\nabla u| \leq 160 \Lambda
$$

which gives a bound on the number $N_{2}:=\#\left\{k: V_{k} \cap \hat{Y} \neq \varnothing\right.$, and $\left.\operatorname{deg}\left(u, V_{k}\right) \neq 0\right\}$, i.e., the number of "charged" $V_{k}$ 's that intersect $\hat{Y}$. In fact, we obtain

$$
N_{2} \leq \sum_{k=1}^{\tilde{N}}\left|\operatorname{deg}\left(u, V_{k}\right)\right| \leq 160 \Lambda
$$


REMARK. We will often refer to a $V_{k}$ such that $\operatorname{deg}\left(u, V_{k}\right) \neq 0$ as a "charged" (or topologically charged) singularity of $u$, and to one such that $\operatorname{deg}\left(u, V_{k}\right)=0$ as a "uncharged" (or neutral or topologically uncharged) singularity of $u$. This terminology is unprecise but helps convey the essential difference between the behavior of $u$ on these two types of sets.

Using this terminology, the charged $V_{k}$ 's that intersect $\hat{Y}$ are $V_{1}, \ldots, V_{N_{2}}$, and the neutral ones are $V_{N_{2}+1}, \ldots, V_{\tilde{N}}$.

\section{The "uncharged" $V_{k}$ 's.}

Although the charged $V_{k}$ 's are the only ones that may change the value of $f(r)=\operatorname{deg}\left(u, S_{r}\right)$, defined in (6.1), in order to prove that these cannot be isolated, we will need some control of $u$ on the uncharged $V_{k}$ 's (i.e., $V_{N_{2}+1}, \ldots, V_{\tilde{N}}$ ), and on the energy minimizing extensions of $u$ to the $W_{k}$ 's that lie inside them. Thus, in this section we will always suppose $k \in\left\{N_{2}+1, \ldots, \tilde{N}\right\}$.

The restriction of $u$ to $V_{k}=\partial W_{k}, g_{k}: V_{k} \longrightarrow S_{\zeta}$, has degree zero (since we are considering only the "uncharged" $V_{k}$ 's) and $W_{k}$ is simply connected, hence $g_{k}$ can be written as

$$
g_{k}=\zeta \exp \left(\imath \theta_{k}\right)
$$

where $\theta_{k}: V_{k} \longrightarrow \mathbb{R}$, is a smooth lifting of $u_{\mid V_{k}}$. For $x \in V_{k}$ we have that

$$
\left|\nabla \theta_{k}\right|^{2}=\left|\nabla\left(\exp \left(\imath \theta_{k}\right)\right)\right|^{2}=\left|\nabla\left(\frac{u}{|u|}\right)\right|^{2} \text {. }
$$

Therefore, by (3.4),

$$
|\nabla u|^{2}=\zeta^{2}\left|\nabla \theta_{k}\right|^{2}+|\nabla| u||^{2},
$$

and, in particular,

$$
\left|\nabla \theta_{k}\right| \leq \frac{|\nabla u|}{\zeta}
$$

As usual, we define the oscillation of $\theta_{k}$ as

$$
\operatorname{osc}\left(\theta_{k}\right):=\sup _{x \in V_{k}}\left(\theta_{k}(x)\right)-\inf _{x \in V_{k}}\left(\theta_{k}(x)\right)
$$


We will prove that the number of $V_{k}$ 's for which $\theta_{k}$ can oscillate considerably, is uniformly bounded (by a constant depending only on the energy bound $\Lambda$ ).

Lemma 2. Given $\Lambda \in \mathbb{R}^{+}$, there is a constant $M \in \mathbb{R}^{+}$such that, for all $\varepsilon>0$, for all $u \in E_{\varepsilon}^{\Lambda}$, if

$$
I:=\left\{k \in\left\{N_{2}+1, \ldots, \tilde{N}\right\}, \text { such that } \operatorname{osc}\left(\theta_{k}\right)>\frac{\pi}{3}\right\},
$$

then,

$$
\# I \leq M=\frac{480 \Lambda}{\pi}
$$

Proof. By the fundamental Theorem of Calculus,

$$
\operatorname{osc}\left(\theta_{k}\right)=\sup _{x, y \in V_{k}}\left(\theta_{k}(x)-\theta_{k}(y)\right) \leq \int_{V_{k}}\left|\frac{\partial \theta_{k}}{\partial \tau}\right| \leq \int_{V_{k}}\left|\nabla \theta_{k}\right| .
$$

Then, using equations (2.10) and (4.3) we obtain

$$
\begin{aligned}
\frac{\pi}{3} \# I & \leq \sum_{k \in I} \operatorname{osc}\left(\theta_{k}\right) \\
& \leq \sum_{k \in I} \int_{V_{k}}\left|\nabla \theta_{k}\right| \\
& \leq \sum_{k \in I} \frac{1}{\zeta} \int_{V_{k}}|\nabla u| \\
& \leq 2 \int_{V(\zeta)}|\nabla u| \\
& \leq 160 \Lambda .
\end{aligned}
$$

Hence,

$$
\# I \leq \frac{3}{\pi} 160 \Lambda=\frac{480}{\pi} \Lambda
$$

Thus, we have proven Lemma 2 with $M=480 \Lambda / \pi$. 
If $\operatorname{deg}\left(u, V_{k}\right)=0$, we know that there exist smooth extensions of $g=u_{\mid V_{k}}: V_{k} \longrightarrow S_{\zeta}$ to $\bar{W}_{k}$, where $S_{\zeta}=\left\{x \in \mathbb{R}^{2}:|x|=\zeta\right\} \simeq S^{1}$, and $W_{k}$ is the domain inside $V_{k}$ (in the sense of Jordan's curve Theorem). Let $H_{g}^{1}:=\left\{u \in H^{1}\left(W_{k}, \mathbb{C}\right): u=g\right.$ on $\left.V_{k}\right\}$. Then, as in the work of F. Bethuel, H. Brezis and F. Hélein [7], we know that

$$
\mu_{g}:=\min _{u \in H_{g}^{1}} E_{\varepsilon}(u)
$$

is achieved by some map $u_{\varepsilon}$, and furthermore, $u_{\varepsilon}$ satisfies the Euler equation

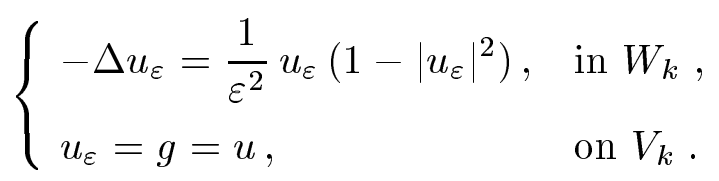

This elliptic system will allow us to prove some sort of maximum principle for $u_{\varepsilon}$ which will give us upper and lower bounds for $\left|u_{\varepsilon}\right|$ in terms of the oscillation of $g=u_{\mid V_{k}}$ or, more precisely, in terms of osc $\left(\theta_{k}\right)$. In particular, we will be able to prove that if the oscillation of $\theta_{k}$ is small enough, then $\left|u_{\varepsilon}\right|$ stays bounded away from zero in $W_{k}$. Together with Lemma 2 this will imply that the number of $W_{k}$ 's for which $\left|u_{\varepsilon}\right|$ can be close to zero, is uniformly bounded.

We start by proving an upper bound for $\left|u_{\varepsilon}\right|$. The following Lemma is just an adaptation of [7, Proposition 2] to our situation.

Lemma 3. Let $u_{\varepsilon}$ be a solution of (4.8). Then, $\left|u_{\varepsilon}\right| \leq 1$, in $W_{k}$.

Proof. We start by observing that

$$
\Delta\left(\left|u_{\varepsilon}\right|^{2}\right)=2 u_{\varepsilon} \Delta u_{\varepsilon}+2\left|\nabla u_{\varepsilon}\right|^{2} .
$$

Hence, by (4.8),

$$
\Delta\left(\left|u_{\varepsilon}\right|^{2}\right)=\frac{2}{\varepsilon^{2}}\left|u_{\varepsilon}\right|^{2}\left(\left|u_{\varepsilon}\right|^{2}-1\right)+2\left|\nabla u_{\varepsilon}\right|^{2} \geq \frac{2}{\varepsilon^{2}}\left|u_{\varepsilon}\right|^{2}\left(\left|u_{\varepsilon}\right|^{2}-1\right) .
$$

Therefore, $v_{\varepsilon}:=\left|u_{\varepsilon}\right|^{2}-1$, will satisfy

$$
\begin{cases}\Delta v_{\varepsilon}-\frac{2}{\varepsilon^{2}}\left|u_{\varepsilon}\right|^{2} v_{\varepsilon} \geq 0, & \text { in } W_{k}, \\ v_{\varepsilon}=\zeta^{2}-1, & \text { on } V_{k}=\partial W_{k} .\end{cases}
$$


Since $-\left(2 / \varepsilon^{2}\right)\left|u_{\varepsilon}\right|^{2} \leq 0$, the maximum principle implies that (see, for instance, [14, Corollary 3.2])

$$
\sup _{W_{k}} v_{\varepsilon} \leq \sup _{V_{k}} v^{+}
$$

where $v^{+}(x):=\max \left\{v_{\varepsilon}(x), 0\right\}$. Hence, since $v^{+}(x):=\max \left\{\zeta^{2}-1,0\right\}=$ 0 , on $V_{k}$, it follows that

$$
\sup _{W_{k}}\left|u_{\varepsilon}\right|^{2}-1=\sup _{W_{k}} v_{\varepsilon} \leq 0
$$

Thus,

$$
\sup _{W_{k}}\left|u_{\varepsilon}\right| \leq 1
$$

This concludes the proof of Lemma 3.

Using this Lemma and equation (4.8), we are now able to obtain

Proposition 1. Suppose that osc $\left(\theta_{k}\right) \leq \pi / 3$. Let $u_{\varepsilon}$ be the minimizer of (4.7). Then,

$$
\left|u_{\varepsilon}(x)\right| \geq \frac{1}{2} \zeta \geq \frac{1}{4}, \quad \text { for all } x \in W_{k} .
$$

Proof. If osc $\left(\theta_{k}\right) \leq \pi / 3$, then $u\left(V_{k}\right)$ is contained in an arch $\hat{\beta}$ of $S_{\zeta}$, of amplitude at most $\pi / 3$. Let $a$ and $b$ be the endpoints of $\hat{\beta}$, and let $B$ be the domain bounded by the straight line $\hat{r}$ passing through $a$ and $b$, and the unit circle $S^{1}$. We claim that the maximum principle implies that

$$
u_{\varepsilon}\left(W_{k}\right) \subset B .
$$

By Lemma 3 we already know that $\left|u_{\varepsilon}\right| \leq 1$, so it suffices to prove that $u_{\varepsilon}\left(W_{k}\right)$ and the origin lie on opposite sides of the straight line $\hat{r}$ defined above.

Choose coordinates $y^{1}, y^{2}$ in the image space such that the $y^{2}$ axis is parallel to $\hat{r}$ (i.e., it is the straight line through the origin parallel to the segment $\overline{a b}$ ), and the $y^{1}$ axis cuts the segment $\overline{a b}$ perpendicularly at its midpoint. In these coordinates we may write

$$
u_{\varepsilon}(x)=\left(\begin{array}{l}
u_{\varepsilon}^{1}(x) \\
u_{\varepsilon}^{2}(x)
\end{array}\right)=\zeta \exp \left(\iota \theta_{k}\right),
$$


where, we are taking the positive $y^{1}$ axis as the origin for the angle $\theta_{k}$.

Since the amplitude $\beta:=\operatorname{osc}\left(\theta_{k}\right) \leq \pi / 3$, the $y^{1}$ coordinate of the endpoints $a$ and $b$ satisfies

$$
\begin{aligned}
\ell & :=y^{1}(a) \\
& =y^{1}(b) \\
& =\min _{x \in V_{k}} y^{1}(u(x)) \\
& =\zeta \cos \left(\frac{\beta}{2}\right) \\
& \geq \zeta \cos \left(\frac{\pi}{6}\right) \\
& =\frac{\zeta}{2} \\
& \geq \frac{1}{4} .
\end{aligned}
$$

On the other hand, since $u_{\varepsilon}$ is a minimizer of $E_{\varepsilon}$, hence a critical point, it is a solution of equation (4.8). In particular $u_{\varepsilon}^{1}$ will satisfy

$$
\begin{cases}-\Delta u_{\varepsilon}^{1}=\frac{1}{\varepsilon^{2}} u_{\varepsilon}^{1}\left(1-\left|u_{\varepsilon}\right|^{2}\right), & \text { in } W_{k}, \\ u_{\varepsilon}^{1} \geq \ell, & \text { on } V_{k}=\partial W_{k} .\end{cases}
$$

Doing a reflection of $u$ across the $y^{2}$ axis in order to make the image lie in the right half-plane, we obtain the map

$$
\tilde{u}_{\varepsilon}(x)=\left(\begin{array}{c}
\tilde{u}_{\varepsilon}^{1}(x) \\
\tilde{u}_{\varepsilon}^{2}(x)
\end{array}\right):=\left(\begin{array}{c}
\left|u_{\varepsilon}^{1}(x)\right| \\
u_{\varepsilon}^{2}(x)
\end{array}\right),
$$

which satisfies

$$
E_{\varepsilon}\left(\tilde{u}_{\varepsilon}\right)=E_{\varepsilon}\left(u_{\varepsilon}\right)=\min _{v \in H_{g}^{1}\left(W_{k}, \mathbb{C}\right)} E_{\varepsilon}(u)
$$

Hence, $\tilde{u}_{\varepsilon}$ is also a minimizer, and thus critical point, of $E_{\varepsilon}$, and therefore, $\tilde{u}_{\varepsilon}^{1}=\left|u_{\varepsilon}^{1}\right|$, satisfies

$$
\begin{cases}-\Delta \tilde{u}_{\varepsilon}^{1}=\frac{1}{\varepsilon^{2}} \tilde{u}_{\varepsilon}^{1}\left(1-\left|u_{\varepsilon}\right|^{2}\right), & \text { in } W_{k}, \\ \tilde{u}_{\varepsilon}^{1} \geq \ell, & \text { on } V_{k}=\partial W_{k} .\end{cases}
$$


Using Lemma 3 we see that the right-hand side of (4.16) is always nonnegative. Hence, $-\Delta \tilde{u}_{\varepsilon} \geq 0$, and thus the maximum principle assures us that

$$
\frac{\min }{\bar{W}_{k}} \tilde{u}_{\varepsilon}^{1}=\min _{V_{k}} \tilde{u}_{\varepsilon}^{1} \geq \ell .
$$

Consequently, using (4.14) we obtain

$$
\min _{\bar{W}_{k}}\left|u_{\varepsilon}^{1}\right| \geq \ell \geq \frac{\zeta}{2} \geq \frac{1}{4} .
$$

Since $u_{\varepsilon}^{1}$ is continuous and $W_{k}$ is connected, $u_{\varepsilon}^{1}\left(W_{k}\right)$ has to be connected. Thus, using (4.17) and the fact that $u_{\varepsilon}^{1}(x) \geq \ell$ on $V_{k}$, we know that we must have

$$
u_{\varepsilon}^{1}(x) \geq \ell, \quad \text { for all } x \in W_{k} .
$$

This, together with equation (4.11), proves claim (4.13). In particular, from (4.18) it follows that

$$
\left|u_{\varepsilon}\right|=\sqrt{\left(u_{\varepsilon}^{1}\right)^{2}+\left(u_{\varepsilon}^{2}\right)^{2}} \geq\left|u_{\varepsilon}^{1}\right| \geq \ell \geq \frac{\zeta}{2} \geq \frac{1}{4}, \quad \text { for all } x \in W_{k},
$$

which is equation (4.12).

REMARK. The same method we used to prove claim (4.13) will give us the slightly more precise result

$$
u_{\varepsilon}\left(W_{k}\right) \subset A \subset B
$$

where $A$ is the closed set bounded by the half-lines $\dot{0} a$ and $\dot{0} b$, the segment $\overline{a b}$ and the circle $S^{1}$. In fact, all we have to do to prove this result is to, instead of using a reflection relative to an axis parallel to the segment $\overline{a b}$, as before, we have to consider reflections with respect to axii which approach $\overline{0 a}$ (and others which approach $\overline{0 b}$ ) on the outside of the set $A$ defined above. 


\section{Blow-up of the energy around an isolated "charged" singu- larity.}

\subsection{The covering argument.}

For simplicity, we will do one more renumbering of the $V_{k}$ 's, $k=$ $1, \ldots, \tilde{N}$ such that

a) $\operatorname{deg}\left(u, V_{k}\right) \neq 0$ and $V_{k} \cap Y \neq \varnothing$ if and only if $k \in\left\{1, \ldots, N_{1}\right\}$.

b) $\operatorname{deg}\left(u, V_{k}\right) \neq 0, V_{k} \cap \hat{Y} \neq \varnothing$ and $V_{k} \cap Y=\varnothing$ if and only if $k \in\left\{N_{1}+1, \ldots, N_{2}\right\}$.

c) $\operatorname{deg}\left(u, V_{k}\right)=0, V_{k} \cap \hat{Y} \neq \varnothing$ and osc $\left(\theta_{k}\right)>\pi / 3$ if and only if $k \in\left\{N_{2}+1, \ldots, N\right\}$.

d) $\operatorname{deg}\left(u, V_{k}\right)=0, V_{k} \cap \hat{Y} \neq \varnothing$ and osc $\left(\theta_{k}\right) \leq \pi / 3$ if and only if $k \in\{N+1, \ldots, \tilde{N}\}$.

From (3.10) it follows that

$$
N_{1} \leq N_{2} \leq 160 \Lambda
$$

On the other hand, Lemma 2 implies that

$$
N=N_{2}+\# I \leq 160 \Lambda+\frac{480}{\pi} \Lambda \leq 320 \Lambda
$$

We remark that (5.2) gives a bound for $N$ which is valid for all $u \in E_{\varepsilon}^{\Lambda}$ and which, moreover, depends only on $\Lambda$ and not on $\varepsilon$. We have no similar bound for $\tilde{N}$, the total number of $V_{k}$ 's that intersect $\hat{Y}$. However, as we will see in this section, a bound on $N$ like (5.2) is enough since Proposition 1 will allow us to prove that the $V_{k}$ 's in condition d) (i.e., those for which $\operatorname{deg}\left(u, V_{k}\right)=0$ and $\operatorname{osc}\left(\theta_{k}\right) \leq \pi / 3$ are "harmless" - in fact, Proposition 1 gives us a good enough control over the behavior of $u$ inside these $V_{k}$ 's for our estimates of lower bounds on the energy of an isolated charged singularity to go through, regardless of the the presence of $V_{k}$ 's of type d) in its neighborhood. We will need the following two rather technical Lemmas to obtain these lower bounds.

The first one is a covering argument that will allow us to see that $W_{1}, \ldots, W_{N}$ can be subdivided into groups, each of which is contained in some ball of radius of order bigger than $\sqrt{\varepsilon}$, and that the different balls are, in some sense, far apart (this type of technique has recently been used by several authors like M. Strüwe or F. Bethuel, H. Brezis 
and F. Hélein or still F.H. Lin in [22] - our approach is closer to that of the latter).

The second Lemma will then serve to prove that if any of the balls $B_{j}$ which intersect $Y$ were charged, then we would have to pay a very high price (of order $|\log \varepsilon|$ ) in energy.

Lemma 4. Fix $\Lambda \in \mathbb{R}^{+}$. Let $u \in E_{\varepsilon}^{\Lambda}$, and $W_{1}, \ldots, W_{N}$ be defined as above. Then, for $\varepsilon$ sufficiently small, there is an integer $m \leq N$, a family of numbers $\alpha_{1}, \ldots, \alpha_{m} \in(1 / 2,1]$, and a family of balls $B_{j}$, $j=1, \ldots, m$, of centers $x_{j}$ and radii $r_{j}$ such that

i) $r_{j} \leq C \varepsilon^{\alpha_{j}}$.

ii) $\bigcup_{i=1}^{N} W_{i} \subset \bigcup_{j=1}^{m} B_{j}$.

iii) The enlarged balls $\tilde{B}_{j}:=B\left(x_{j}, \varepsilon^{-\alpha_{j} /\left(2^{N+1}+1\right)} r_{j}\right)$ are pairwise disjoint.

Proof. We have fixed $\Lambda \in \mathbb{R}^{+}$, and we are looking at maps $u \in E_{\varepsilon}^{\Lambda}$, for $\varepsilon$ sufficiently small (to be chosen later). We define $W_{1}, \ldots, W_{N}$ as above (thus they will be open, simply-connected subsets of $\Omega \subset \mathbb{R}^{2}$, such that $\partial W_{k}=V_{k}$ ). By equation (5.2) we know that there exists a uniform bound on $N$ depending only on the energy level $\Lambda$ we are considering, and not on $\varepsilon$ - to be able to change $\varepsilon$ while having an uniform bound on the number $m$ of balls used in the covering is crucial for our argument to work.

On the other hand, by (2.12) we have that

$$
\operatorname{diam}\left(W_{k}\right) \leq \frac{1}{2} \mathcal{H}^{1}\left(V_{k}\right) \leq 160 \Lambda \varepsilon
$$

Hence our Lemma follows from the more general covering argument stated in Lemma 7 of the Appendix. In fact, it corresponds to the special case where $C=160 \Lambda$ and $\alpha=1$.

\subsection{Lower-bound for the energy around an isolated charged singularity.}

Lemma 5. Let $R_{1}, R_{2} \in \mathbb{R}^{+}$be such that $R_{1}<R_{2}$. Let $\Omega$ be the annulus $\Omega=B\left(0, R_{2}\right) \backslash B\left(0, R_{1}\right)$, and $u \in H^{1}(\Omega, \mathbb{C})$ be such that exists 
$\sigma \in \mathbb{R}^{+}$such that $|u(x)| \geq \sigma>0$, for all $x \in \Omega$, and $\operatorname{deg}\left(u, S_{R_{1}}\right)=$ $\operatorname{deg}\left(u, S_{R_{2}}\right)=d \neq 0$. Then,

$$
E_{\varepsilon}(u) \geq \pi d^{2} \sigma^{2} \log \left(\frac{R_{2}}{R_{1}}\right) .
$$

Proof. We have that

$$
E_{\varepsilon}(u) \geq \frac{1}{2} \int_{\Omega}|\nabla u|^{2}, \quad \text { for all } u \in H^{1}(\Omega, \mathbb{C}) .
$$

Hence, we will concentrate on obtaining a lower bound for the Dirichlet energy of $u$ (the right hand side of (5.5)). Since, by hypothesis, $|u| \geq$ $\sigma>0$, we may define

$$
v:=\frac{u}{|u|} \in H^{1}\left(\Omega, S^{1}\right), \text { and } \operatorname{deg}\left(v, S_{R_{1}}\right)=\operatorname{deg}\left(v, S_{R_{2}}\right)=d \neq 0 .
$$

By (3.5) we know that

$$
|\nabla u|^{2} \geq|u|^{2}|\nabla v|^{2} \geq \sigma^{2}|\nabla v|^{2}
$$

We define

$$
\mathcal{V}_{d}=\left\{v \in H^{1}\left(\Omega, S^{1}\right): \operatorname{deg}\left(v, S_{R_{1}}\right)=\operatorname{deg}\left(v, S_{R_{2}}\right)=d\right\} .
$$

From (5.5) and (5.6) it follows that

$$
E_{\varepsilon}(u) \geq \frac{1}{2} \int|\nabla u|^{2} \geq \sigma^{2} \inf _{v \in \mathcal{V}_{d}}\left(\frac{1}{2} \int|\nabla v|^{2}\right) .
$$

The problem of determining

$$
\inf _{v \in \mathcal{V}_{d}}\left(\frac{1}{2} \int|\nabla u|^{2}\right)
$$

has already been extensively studied. In fact we can reduce it, using an associated linear problem (see, for instance, [8, Theorems I.1 and II.1, and their Corollaries]), to determining the Dirichlet energy of a harmonic map $\Phi$ such that

$$
\begin{cases}\Delta \Phi=0, & \text { in } \Omega \\ \Phi=0, & \text { on } S_{R_{2}} \\ \Phi=C, & \text { on } S_{R_{1}} \\ \int_{S_{R_{i}}} \frac{\partial \Phi}{\partial \nu}=2 \pi d, & \end{cases}
$$


where $C$ is some constant, and $\nu$ is the outward normal to $B_{R_{1}}$ and also the outward normal to $B_{R_{2}}$ (so $\nu$ will point inside $\Omega$ on $S_{R_{1}}$ and outside on $S_{R_{2}}$ ).

We can easily check that $\Phi=d \log \left(r / R_{2}\right)$ is a solution of (5.8). Therefore, by the proof of [8, Theorem I.1] (see step 1 of that proof it is essentially a consequence of Poincaré's Lemma) we know that for all $v \in H^{1}\left(\Omega, S^{1}\right): \operatorname{deg}\left(v, S_{R_{i}}\right)=d, i=1,2$,

$$
\begin{aligned}
\int_{\Omega}|\nabla v|^{2} & \geq \int_{\Omega}|\nabla \Phi|^{2} \\
& =\int_{\Omega}\left|\frac{d}{r}\right|^{2} \\
& =\int_{0}^{2 \pi} d \theta \int_{R_{1}}^{R_{2}} r \frac{d^{2}}{r^{2}} d r \\
& =2 \pi d^{2} \log \left(\frac{R_{2}}{R_{1}}\right) .
\end{aligned}
$$

Combining equations (5.7) and (5.9) we obtain

$$
E_{\varepsilon}(u) \geq \pi \sigma^{2} d^{2} \log \left(\frac{R_{2}}{R_{1}}\right),
$$

which is the desired result.

We are now ready to prove the main result of this section.

Theorem 8. Let $\Lambda \in \mathbb{R}$ be fixed and $u \in E_{\varepsilon}^{\Lambda}$. Then, there exists $\varepsilon_{0}>0$ (depending only on $\Lambda$ ) such that if $\varepsilon<\varepsilon_{0}$, then $B_{j} \cap Y \neq \varnothing$ implies that $\operatorname{deg}\left(u, \partial B_{j}\right)=0$, where the balls $B_{j}$ are given by Lemma 4 .

Proof. Suppose that for some $\varepsilon$, sufficiently small to apply Lemma 4, there exists $u \in E_{\varepsilon}^{\Lambda}$ such that in Lemma 4 we obtained a ball $B_{j}$ such that $B_{j} \cap Y \neq \varnothing$ and $\operatorname{deg}\left(u, \partial B_{j}\right) \neq 0$. Since $B_{j} \cap Y \neq \varnothing$, if $\varepsilon$ is sufficiently small (depending only on $\Lambda$ ) $\tilde{B}_{j} \subset \hat{Y}$ (because the radius of $\tilde{B}_{j}$ tends to zero when $\left.\varepsilon \longrightarrow 0\right)$. Thus, since in the covering argument we took care of all the $V_{k}$ 's such that $V_{k} \cap \hat{Y} \neq \varnothing$ and $\operatorname{deg}\left(u, V_{j}\right) \neq 0$ or $\operatorname{osc}\left(\theta_{k}\right)>\pi / 3$, we know that the annulus $D_{j}:=\tilde{B}_{j} \backslash B_{j}$ may only intersect uncharged $V_{k}$ 's such that osc $\left(\theta_{k}\right) \leq \pi / 3$ (what we called $V_{k}$ 's of type d) in the beginning of this section). 
We may suppose, without loss of generality, that the $V_{k}$ 's that intersect $D_{j}$ are $V_{N+1}, \ldots, V_{\hat{N}}$, for some $\hat{N} \leq \tilde{N}$. We know that $\operatorname{osc}\left(\theta_{k}\right) \leq \pi / 3, k=N+1, \ldots, \hat{N}$. However, we cannot apply Lemma 5 directly to $u$ on $D_{j}$ since a priori we have no lower bound on $|u|$ inside $W_{N+1}, \ldots, W_{\hat{N}}$. Nevertheless, if we replace $u$ inside each of the $W_{k}$, $k=N+1, \ldots, \hat{N}$, by the corresponding minimizer of (4.7), we will decrease the energy and, at the same time, by Proposition 1, we will have a lower bound on the absolute value of the map obtained. Let

$$
\tilde{u}:=\left\{\begin{array}{l}
u, \quad \text { in } D_{j} \backslash \bigcup_{k=N+1}^{\hat{N}} W_{k}, \\
u_{\varepsilon}, \quad \text { in } W_{k}, k=N+1, \ldots, \hat{N},
\end{array}\right.
$$

where $u_{\varepsilon}$ is the minimizer of $E_{\varepsilon}$ in $W_{k}$ with boundary value $u$. In particular, $u_{\varepsilon}$ satisfies equation (4.8). By construction, $|\tilde{u}| \geq \zeta \geq 1 / 2$ in $D_{j} \backslash \bigcup_{k=N+1}^{\hat{N}} W_{k}$, and by Proposition $1,|\tilde{u}|=\left|u_{\varepsilon}\right| \geq 1 / 4$ in $W_{k}$, $k=N+1, \ldots, \hat{N}$. Therefore,

$$
|\tilde{u}| \geq \frac{1}{4}, \quad \text { in } D_{j}
$$

Hence, $\operatorname{deg}\left(\tilde{u}, \partial \tilde{B}_{j}\right)=\operatorname{deg}\left(\tilde{u}, \partial B_{j}\right)=d \neq 0$.Thus, we may apply Lemma 5 to $\tilde{u}$ in $D_{j}$. Denoting the energy of a map $w$ in a domain $G$ by

$$
E_{\varepsilon}(w, G):=\frac{1}{2} \int_{G}|\nabla w|^{2}+\frac{1}{4 \varepsilon^{2}} \int_{G}\left(1-|w|^{2}\right)^{2},
$$

this Lemma yields

$$
E_{\varepsilon}\left(\tilde{u}, D_{j}\right) \geq \pi d^{2}\left(\frac{1}{4}\right)^{2} \log \left(\varepsilon^{-\alpha_{j} /\left(2^{N+1}+1\right)}\right) .
$$

Since $\alpha_{j} \geq 1 / 2$ (by Lemma 4 ), we have that

$$
E_{\varepsilon}\left(\tilde{u}, D_{j}\right) \geq \frac{\pi d^{2}}{16} \log \left(\varepsilon^{-1 /\left(2\left(2^{N+1}+1\right)\right)}\right)=-\frac{\pi d^{2}}{32\left(2^{N+1}+1\right)} \log \varepsilon .
$$

We claim that, for $\varepsilon$ sufficiently small

$$
E_{\varepsilon}(u, \Omega) \geq E_{\varepsilon}\left(\tilde{u}, D_{j}\right)
$$


ProOF OF CLAim (5.14). We have that

$$
\begin{aligned}
E_{\varepsilon}\left(\tilde{u}, D_{j}\right) & =E_{\varepsilon}\left(\tilde{u}, D_{j} \backslash \bigcup_{k=N+1}^{\hat{N}} W_{k}\right)+\sum_{k=N+1}^{\hat{N}} E_{\varepsilon}\left(\tilde{u}, W_{k} \cap D_{j}\right) \\
& \leq E_{\varepsilon}\left(\tilde{u}, D_{j} \backslash \bigcup_{k=N+1}^{\hat{N}} W_{k}\right)+\sum_{k=N+1}^{\hat{N}} E_{\varepsilon}\left(\tilde{u}, W_{k}\right) .
\end{aligned}
$$

By construction, $\tilde{u}=u$ on $D_{j} \backslash \bigcup_{k=N+1}^{\hat{N}} W_{k}$, we have that

$$
E_{\varepsilon}\left(\tilde{u}, D_{j} \backslash \bigcup_{k=N+1}^{\hat{N}} W_{k}\right)=E_{\varepsilon}\left(u, D_{j} \backslash \bigcup_{k=N+1}^{\hat{N}} W_{k}\right)
$$

and, on the other hand, by the definition of $u_{\varepsilon}$ as the minimizer of (4.7), we also have that

$$
E_{\varepsilon}\left(\tilde{u}, W_{k}\right) \leq E_{\varepsilon}\left(u, W_{k}\right), \quad \text { for } k=N+1, \ldots, \hat{N}
$$

Therefore, it follows from (5.15) that

$$
\begin{aligned}
E_{\varepsilon}\left(\tilde{u}, D_{j}\right) & \leq E_{\varepsilon}\left(u, D_{j} \backslash \bigcup_{k=N+1}^{\hat{N}} W_{k}\right)+\sum_{k=N+1}^{\hat{N}} E_{\varepsilon}\left(u, W_{k}\right) \\
& =E_{\varepsilon}\left(u, D_{j} \cup W_{N+1} \cup \cdots \cup W_{\hat{N}}\right) \\
& \leq E_{\varepsilon}(u, \Omega)
\end{aligned}
$$

since $W_{k} \subset \hat{Y} \subset \Omega, k=N+1, \ldots, \hat{N}$, if $\varepsilon$ is sufficiently small. This concludes the proof of claim (5.14).

Combining (5.13) and (5.14) we have that for $\varepsilon$ sufficiently small,

$$
E_{\varepsilon}(u, \Omega) \geq-\frac{\pi d^{2}}{32\left(2^{N+1}+1\right)} \log \varepsilon \geq C d^{2}|\log \varepsilon|,
$$

where $C$ is a positive constant only depending on $\Lambda$ (in fact, using equation (5.2) we may choose $\left.C=\pi /\left(32\left(2^{320 \Lambda+1}+1\right)\right)>0\right)$.

If, as we supposed, $d \neq 0$, then, since $u \in E_{\varepsilon}^{\Lambda}$, we would have that $C d^{2}|\log \varepsilon| \leq \Lambda$, for all $\varepsilon$ sufficiently small. However, this is clearly not 
true for $\varepsilon \leq \exp \left(-\Lambda /\left(C d^{2}\right)\right)$. Hence, $d$ must be zero, which concludes the proof of Theorem 8 .

REMARK. Theorem 8 proves rigorously our idea that as $\varepsilon$ gets small the charged $V_{k}$ 's have to cluster, giving rise to "neutral" (deg $\left.=0\right) B_{j}$ 's, or to "drift" towards the boundary $\partial \Omega$ (thus exiting the interior domain $Y)$. Hence, in the interior of $\Omega$, and for a distance scale of order $\varepsilon^{1 / 2}$, the charged singularities shouldn't be "perceptible".

\section{Definition of the degree of $u$ in $\Omega$.}

In this section we define the degree of $u$ in $\Omega$, which is an integer, and show that this integer is well defined.

Let

$$
v:=\frac{u}{|u|}: \hat{Y} \backslash \bigcup_{k=1}^{\tilde{N}} W_{k} \longrightarrow S^{1}
$$

and

$$
A:=\left\{r \in\left(\frac{1}{2}, \frac{3}{4}\right): S_{r} \cap V_{k}=\varnothing, \text { for all } k=1, \ldots, \tilde{N}\right\} .
$$

As before, for $r \in A$, we define

$$
f(r):=\frac{1}{2 \pi} \int_{S_{r}} v \times \frac{\partial v}{\partial \tau}=\operatorname{deg}\left(u, S_{r}\right),
$$

and we define the approximate degree as

$$
\operatorname{adeg}(u):=\frac{1}{2 \pi|A|} \int_{A} \int_{S_{r}} v \times \frac{\partial v}{\partial \tau} d \tau d r=\frac{1}{|A|} \int_{A} f(r) d r .
$$

The function $f$ may only change value when we cross a charged $V_{k}$ since if $r_{1}, r_{2} \in A, r_{2}>r_{1}$, then

$$
\begin{gathered}
f\left(r_{2}\right)-f\left(r_{1}\right)=\sum_{k \in I_{r_{1}, r_{2}}} \operatorname{deg}\left(u, V_{k}\right), \\
I_{r_{1}, r_{2}}=\left\{k: V_{k} \subset B\left(0, r_{2}\right) \backslash B\left(0, r_{1}\right)\right\} .
\end{gathered}
$$

By (5.3), (5.2), Lemma 4 and Theorem 8 , inside $Y$ we can cover all the charged $V_{k}$ 's by an uniformly bounded number of balls $B_{1}, \ldots, B_{m}$, 
with $m \leq 320 \Lambda$, and such that $r_{j}=\operatorname{radius}\left(B_{j}\right) \leq 160 \Lambda \varepsilon^{1 / 2}$, and $\operatorname{deg}\left(u, \partial B_{j}\right)=0$. Hence the function $f$ will always have the same value in $\breve{A}:=A \backslash B$, where $B:=\bigcup_{j=1}^{m}\left\{r: S_{R} \cap B_{j} \neq \varnothing\right\}$. This is the value we use to define $\operatorname{deg}(u, \Omega) \in \mathbb{Z}$.

When $\varepsilon \longrightarrow 0$ the approximate degree $(\operatorname{adeg}(u))$ approaches this value. In fact, from (5.2) and Lemma 4 , it follows that

$$
|B| \leq 2 \sum_{j=1}^{m} r_{j} \leq 2 m 160 \Lambda \varepsilon^{1 / 2} \leq(320 \Lambda)^{2} \varepsilon^{1 / 2} .
$$

Furthermore, even inside $A \cap B$ the value of $f(r)=\operatorname{deg}\left(u, S_{r}\right)$ is uniformly bounded - equations (3.9) and (6.3) imply that

$$
|f-\operatorname{deg}(u, \Omega)| \leq \sum_{k=1}^{N_{1}}\left|\operatorname{deg}\left(u, V_{k}\right)\right| \leq 160 \Lambda .
$$

Thus, using (2.7), (6.4) and (6.5), we obtain

$$
\begin{aligned}
|\operatorname{adeg}(u)-\operatorname{deg}(u)| & =\left|\frac{1}{|A|} \int_{A} f(r) d r-\frac{1}{|A|} \int_{A} \operatorname{deg}(u, \Omega) d r\right| \\
& \leq \frac{1}{|A|} \int_{A}|f(r)-\operatorname{deg}(u, \Omega)| \\
& \leq \frac{1}{|A|}|B| 160 \Lambda \\
& \leq \frac{(320 \Lambda)^{3}}{2\left(\frac{1}{4}-\mathcal{H}^{1}(V(\zeta))\right)} \varepsilon^{1 / 2} \\
& \leq \frac{(320 \Lambda)^{3}}{\frac{1}{2}-320 \Lambda \varepsilon} \varepsilon^{1 / 2} .
\end{aligned}
$$

Since this bound depends only on $\Lambda$ and $\varepsilon$ (and not on $u$ ), we will have that $\operatorname{adeg}(u)$ will converge to $\operatorname{deg}(u, \Omega) \in \mathbb{Z}$, uniformly in $u \in E_{\varepsilon}^{\Lambda}$. Hence, given $\Lambda$, we know that for $\varepsilon$ sufficiently small

$$
|\operatorname{adeg}(u)-\operatorname{deg}(u)| \leq \frac{1}{4}
$$

and therefore, the knowledge of $\operatorname{adeg}(u)$ will determine the integer $\operatorname{deg}(u)$ as desired. 
REMARK. Of course we can also obtain $\operatorname{deg}(u, \Omega)$ by evaluating $f(r)=$ $\operatorname{deg}\left(u, S_{r}\right)$ for any $r \in \breve{A}=A \backslash B$. The problem is that the process of obtaining the balls $B_{j}$ that define $B$ is very elaborate - hence our choice of also showing how to obtain $\operatorname{deg}(u, \Omega)$ using the approximate degree. We remark also that the $B_{j}$ 's obtained using Lemma 4 , and thus also $B$, are not uniquely determined. However, using estimate (6.4), it is easy to check that (for sufficiently small $\varepsilon$, as usual) the value of $\operatorname{deg}(u, \Omega)$ obtained by evaluating $f(r)$ in $\breve{A}$, is independent of the particular $B_{j}$ 's used in the process.

\section{Continuity of $\operatorname{deg}(u, \Omega)$.}

This section is devoted to showing that the notion of $\operatorname{deg}(u, \Omega)$ we defined in the previous section (Section 6) is continuous in $H^{1}(\Omega)$ topology inside each level set of the Ginzburg-Landau energy (1.1). This result will be stated in Theorem 9 at the end of the section.

Let $\Lambda \in \mathbb{R}^{+}$be given and $\varepsilon<\varepsilon_{0}$ (with $\varepsilon_{0}$ defined as in Theorem 8) and consider $u_{1}, u_{2} \in E_{\varepsilon}^{\Lambda}$. Suppose $B_{1}^{i}, \ldots, B_{m_{i}}^{i}$, are the balls obtained when applying Lemma 4 to $u_{i}, i=1,2$, and $V_{k}^{i}, k=1, \ldots, \tilde{N}_{i}, i=1,2$, denote the corresponding $V_{k}$ 's. We define, as before, $v_{i}:=u_{i} /\left|u_{i}\right|$,

$$
\begin{gathered}
A_{i}:=\left\{r \in\left(\frac{1}{2}, \frac{3}{4}\right): S_{r} \cap V_{k}^{i}=\varnothing, \text { for all } k=1, \ldots, \tilde{N},\right. \\
\text { and } \left.S_{r} \cap B_{j}^{i}=\varnothing, \text { for all } j=1, \ldots, m_{i}\right\}, \\
f_{i}(r):=\frac{1}{2 \pi} \int_{S_{r}} v_{i} \times \frac{\partial v_{i}}{\partial \tau} d \tau, \quad \text { for } r \in A_{i} .
\end{gathered}
$$

Then, denoting $A:=A_{1} \cap A_{2}$,

$$
\operatorname{deg}\left(u_{i}, \Omega\right)=\frac{1}{\left|A_{i}\right|} \int_{A_{i}} f_{i}(r) d r=\frac{1}{|A|} \int_{A} f_{i}(r) d r
$$

since $f_{i}(r)=C^{t e}=\operatorname{deg}\left(u_{i}, \Omega\right)$ in $A_{i}$ (hence also in $\left.A \subset A_{i}\right)$. Therefore, denoting $G:=\{(r, \theta): r \in A, \theta \in[0,2 \pi)\}$,

$$
\left|\operatorname{deg}\left(u_{1}, \Omega\right)-\operatorname{deg}\left(u_{2}, \Omega\right)\right|
$$

$$
=\frac{1}{2 \pi|A|}\left|\int_{A} \int_{S_{r}}\left(\frac{u_{1}}{\left|u_{1}\right|} \times \partial_{\tau}\left(\frac{u_{1}}{\left|u_{1}\right|}\right)-\frac{u_{2}}{\left|u_{2}\right|} \times \partial_{\tau}\left(\frac{u_{2}}{\left|u_{2}\right|}\right)\right) d \tau d r\right|
$$




$$
=\frac{1}{2 \pi|A|}\left|\int_{A} \int_{S_{r}}\left(\frac{u_{1}}{\left|u_{1}\right|^{2}} \times \frac{\partial u_{1}}{\partial \tau}-\frac{u_{2}}{\left|u_{2}\right|^{2}} \times \frac{\partial u_{2}}{\partial \tau}\right) d \tau d r\right|,
$$

since

$$
\begin{aligned}
\frac{u_{i}}{\left|u_{i}\right|} \times \partial_{\tau}\left(\frac{u_{i}}{\left|u_{i}\right|}\right) & =\frac{u_{i}}{\left|u_{i}\right|} \times\left(\frac{1}{\left|u_{i}\right|} \frac{\partial u_{i}}{\partial \tau}\right)+\frac{u_{i}}{\left|u_{i}\right|} \times\left(u_{i} \partial_{\tau}\left(\frac{1}{\left|u_{i}\right|}\right)\right) \\
& =\frac{u_{i}}{\left|u_{i}\right|} \times\left(\frac{1}{\left|u_{i}\right|} \frac{\partial u_{i}}{\partial \tau}\right),
\end{aligned}
$$

because $u_{i} \times u_{i}=0$.

Furthermore, from equation (2.11) and Lemma 4, it follows that $\left|A_{1}\right|,\left|A_{2}\right|$ and $|A| \longrightarrow 1 / 4$ uniformly when $\varepsilon \longrightarrow 0$, and thus, in particular, we have that for $\varepsilon$ sufficiently small (independent of the particular choice of $\left.u_{1}, u_{2} \in E_{\varepsilon}^{\Lambda}\right),|A|>1 /(2 \pi)$. Hence, equation (7.2) yields that for all $\varepsilon$ as above,

$$
\begin{aligned}
\left|\operatorname{deg}\left(u_{1}, \Omega\right)-\operatorname{deg}\left(u_{2}, \Omega\right)\right| & \\
& =\frac{1}{2 \pi|A|}\left|\int_{A} \int_{S_{r}}\left(\frac{u_{1}}{\left|u_{1}\right|^{2}} \times \frac{\partial u_{1}}{\partial \tau}-\frac{u_{2}}{\left|u_{2}\right|^{2}} \times \frac{\partial u_{2}}{\partial \tau}\right) d \tau d r\right| \\
& \leq \frac{1}{2 \pi|A|} \int_{G}\left|\frac{u_{1}}{\left|u_{1}\right|^{2}} \times \frac{\partial u_{1}}{\partial \tau}-\frac{u_{2}}{\left|u_{2}\right|^{2}} \times \frac{\partial u_{2}}{\partial \tau}\right| \\
& \leq\left\|\frac{u_{1}}{\left|u_{1}\right|^{2}} \times \frac{\partial u_{1}}{\partial \tau}-\frac{u_{2}}{\left|u_{2}\right|^{2}} \times \frac{\partial u_{2}}{\partial \tau}\right\|_{L^{1}(G)} \cdot
\end{aligned}
$$

We can write the integrand in (7.3) as

$$
\begin{aligned}
& \frac{u_{1}}{\left|u_{1}\right|^{2}} \times \frac{\partial u_{1}}{\partial \tau}-\frac{u_{2}}{\left|u_{2}\right|^{2}} \times \frac{\partial u_{2}}{\partial \tau} \\
& \quad=\frac{1}{\left|u_{1}\right|} \frac{u_{1}}{\left|u_{1}\right|} \times \frac{\partial u_{1}}{\partial \tau}-\frac{1}{\left|u_{2}\right|} \frac{u_{2}}{\left|u_{2}\right|} \times \frac{\partial u_{2}}{\partial \tau} \\
& \quad=\left(\frac{1}{\left|u_{1}\right|}-\frac{1}{\left|u_{2}\right|}\right) \frac{u_{1}}{\left|u_{1}\right|} \times \frac{\partial u_{1}}{\partial \tau}-\frac{1}{\left|u_{2}\right|}\left(\frac{u_{1}}{\left|u_{1}\right|} \times \frac{\partial u_{1}}{\partial \tau}-\frac{u_{2}}{\left|u_{2}\right|} \times \frac{\partial u_{2}}{\partial \tau}\right) .
\end{aligned}
$$

Moreover, one can write the last factor in (7.4) as

$$
\frac{u_{1}}{\left|u_{1}\right|} \times \frac{\partial u_{1}}{\partial \tau}-\frac{u_{2}}{\left|u_{2}\right|} \times \frac{\partial u_{2}}{\partial \tau}
$$




$$
\begin{aligned}
= & \frac{1}{\left|u_{1}\right|}\left(u_{1} \times \frac{\partial u_{1}}{\partial \tau}-u_{2} \times \frac{\partial u_{2}}{\partial \tau}\right)+\left(\frac{1}{\left|u_{1}\right|}-\frac{1}{\left|u_{2}\right|}\right) u_{2} \times \frac{\partial u_{2}}{\partial \tau} \\
= & \frac{1}{\left|u_{1}\right|}\left(\left(u_{1}-u_{2}\right) \times \frac{\partial u_{1}}{\partial \tau}+u_{2} \times \frac{\partial\left(u_{1}-u_{2}\right)}{\partial \tau}\right) \\
& +\left(\frac{1}{\left|u_{1}\right|}-\frac{1}{\left|u_{2}\right|}\right) u_{2} \times \frac{\partial u_{2}}{\partial \tau} .
\end{aligned}
$$

From (7.4) and (7.5) it follows that

$$
\begin{aligned}
& \frac{u_{1}}{\left|u_{1}\right|^{2}} \times \frac{\partial u_{1}}{\partial \tau}-\frac{u_{2}}{\left|u_{2}\right|^{2}} \times \frac{\partial u_{2}}{\partial \tau} \\
& =\left(\frac{1}{\left|u_{1}\right|}-\frac{1}{\left|u_{2}\right|}\right) \frac{u_{1}}{\left|u_{1}\right|} \times \frac{\partial u_{1}}{\partial \tau}+\frac{1}{\left|u_{1}\right|\left|u_{2}\right|}\left(\left(u_{1}-u_{2}\right) \times \frac{\partial u_{1}}{\partial \tau}\right) \\
& \quad+\frac{1}{\left|u_{1}\right|}\left(\frac{u_{2}}{\left|u_{2}\right|} \times \frac{\partial\left(u_{1}-u_{2}\right)}{\partial \tau}\right)+\left(\frac{1}{\left|u_{1}\right|}-\frac{1}{\left|u_{2}\right|}\right)\left(u_{2} \times \frac{\partial u_{2}}{\partial \tau}\right) .
\end{aligned}
$$

On the other hand, since $\left|u_{i}\right| \geq 1 / 2$ in $G$, we have that

$$
\frac{1}{\left|u_{i}\right|} \leq 2, i=1,2 \text {, and } \frac{1}{\left|u_{1}\right|\left|u_{2}\right|} \leq 4 \text {, in } G \text {. }
$$

Furthermore, we have the following estimates for $v_{i}=u_{i} /\left|u_{i}\right|$,

$$
\left\|\frac{u_{i}}{\left|u_{i}\right|}\right\|_{L^{\infty}(\Omega)}=1
$$

(7.9) $\left\|\frac{u_{i}}{\left|u_{i}\right|}\right\|_{L^{2}(G)} \leq\left\|\frac{u_{i}}{\left|u_{i}\right|}\right\|_{L^{\infty}(G)}|G|^{1 / 2} \leq|G|^{1 / 2} \leq|Y|^{1 / 2}=\frac{\sqrt{5 \pi}}{4}$.

Regarding the tangential derivatives, we have that $\left|\partial u_{i} / \partial \tau\right| \leq\left|\nabla u_{i}\right|$, and thus,

$$
\left\|\frac{\partial u_{i}}{\partial \tau}\right\|_{L^{2}(G)} \leq\left\|\nabla u_{i}\right\|_{L^{2}(G)} \leq\left\|\nabla u_{i}\right\|_{L^{2}(\Omega)}
$$

and also that

$$
\left|\frac{\partial\left(u_{1}-u_{2}\right)}{\partial \tau}\right| \leq\left|\nabla\left(u_{1}-u_{2}\right)\right|
$$

which implies that

$(7.11)\left\|\frac{\partial\left(u_{1}-u_{2}\right)}{\partial \tau}\right\|_{L^{2}(G)} \leq\left\|\nabla\left(u_{1}-u_{2}\right)\right\|_{L^{2}(G)} \leq\left\|\nabla\left(u_{1}-u_{2}\right)\right\|_{L^{2}(\Omega)}$. 
Finally, we can easily check that

$$
\left|\frac{1}{\left|u_{1}\right|}-\frac{1}{\left|u_{2}\right|}\right|=\frac{|| u_{1}|-| u_{2}||}{\left|u_{1}\right|\left|u_{2}\right|} \leq \frac{\left|u_{1}-u_{2}\right|}{\left|u_{1}\right|\left|u_{2}\right|} \leq 4\left|u_{1}-u_{2}\right|,
$$

which, in turn, yields

$$
\left\|\frac{1}{\left|u_{1}\right|}-\frac{1}{\left|u_{2}\right|}\right\|_{L^{2}(G)} \leq 4\left\|u_{1}-u_{2}\right\|_{L^{2}(G)} \leq 4\left\|u_{1}-u_{2}\right\|_{L^{2}(\Omega)} .
$$

Moreover, since we supposed that $u_{i} \in E_{\varepsilon}^{\Lambda}$, we have, as in (2.5),

$$
\left\|\nabla u_{i}\right\|_{L^{2}(G)} \leq\left\|\nabla u_{i}\right\|_{L^{2}(\Omega)} \leq \sqrt{2 E_{\varepsilon}\left(u_{i}\right)} \leq \sqrt{2 \Lambda}
$$

Using the Cauchy-Schwarz inequality and equations (7.6), (7.7), (7.8), (7.9), (7.10), (7.11), (7.12) and (7.13), it follows from equation (7.3) that

$$
\begin{aligned}
&\left|\operatorname{deg}\left(u_{1}, \Omega\right)-\operatorname{deg}\left(u_{2}, \Omega\right)\right| \\
& \leq\left\|\frac{u_{1}}{\left|u_{1}\right|^{2}} \times \frac{\partial u_{1}}{\partial \tau}-\frac{u_{2}}{\left|u_{2}\right|^{2}} \times \frac{\partial u_{2}}{\partial \tau}\right\|_{L^{1}(G)} \\
& \leq\left\|\frac{u_{1}}{\left|u_{1}\right|}\right\|_{L^{\infty}(G)}\left\|\frac{1}{\left|u_{1}\right|}-\frac{1}{\left|u_{2}\right|}\right\|_{L^{2}(G)}\left\|\nabla u_{1}\right\|_{L^{2}(G)} \\
&+4\left\|u_{1}-u_{2}\right\|_{L^{2}(G)}\left\|\nabla u_{1}\right\|_{L^{2}(G)} \\
&+2\left\|\frac{u_{2}}{\left|u_{2}\right|}\right\|_{L^{2}(G)}\left\|\nabla\left(u_{1}-u_{2}\right)\right\|_{L^{2}(G)} \\
&+\left\|\frac{u_{2}}{\left|u_{2}\right|}\right\|_{L^{\infty}(G)}\left\|\frac{1}{\left|u_{1}\right|}-\frac{1}{\left|u_{2}\right|}\right\|_{L^{2}(G)}\left\|\nabla u_{2}\right\|_{L^{2}(G)} \\
& \leq 4\left\|\nabla u_{1}\right\|_{L^{2}(G)}\left\|u_{1}-u_{2}\right\|_{L^{2}(G)}+4\left\|\nabla u_{1}\right\|_{L^{2}(G)}\left\|u_{1}-u_{2}\right\|_{L^{2}(G)} \\
&+2|Y|^{1 / 2}\left\|\nabla\left(u_{1}-u_{2}\right)\right\|_{L^{2}(G)}+4\left\|\nabla u_{2}\right\|_{L^{2}(G)}\left\|u_{1}-u_{2}\right\|_{L^{2}(G)} \\
& \leq\left(8\left\|\nabla u_{1}\right\|_{L^{2}(\Omega)}+4\left\|\nabla u_{2}\right\|_{L^{2}(\Omega)}\right)\left\|u_{1}-u_{2}\right\|_{L^{2}(\Omega)} \\
&+2 \frac{\sqrt{5 \pi}}{4}\left\|\nabla\left(u_{1}-u_{2}\right)\right\|_{L^{2}(\Omega)} \\
& \leq 12 \sqrt{2 \Lambda}\left\|u_{1}-u_{2}\right\|_{L^{2}(\Omega)}+\frac{\sqrt{5 \pi}}{2}\left\|\nabla\left(u_{1}-u_{2}\right)\right\|_{L^{2}(\Omega)} \\
& \leq C\left\|u_{1}-u_{2}\right\|_{H^{1}(\Omega)},
\end{aligned}
$$


where $C$ is a constant that depends only on the energy bound $\Lambda$ (we may take $C=12 \sqrt{2 \Lambda}+\sqrt{5 \pi} / 2)$. Therefore, we have proven the following Theorem which is the main result of this section.

Theorem 9. Let $\Lambda>0$ be given and $\varepsilon$ be sufficiently small. Then, inside the level set $E_{\varepsilon}^{\Lambda}$ the degree defined as above is continuous in $H^{1}(\Omega)$ topology, and there is a constant $C$, depending only on $\Lambda$, such that for all $u_{1}, u_{2} \in E_{\varepsilon}^{\Lambda}$

$$
\left|\operatorname{deg}\left(u_{1}, \Omega\right)-\operatorname{deg}\left(u_{2}, \Omega\right)\right| \leq C\left\|u_{1}-u_{2}\right\|_{H^{1}(\Omega)} .
$$

\section{Proof of Theorem 1 and Theorem 6.}

We start by proving Theorem 1 , i.e. the case where $\Omega$ is of the special form we studied (the annulus $\Omega=\left\{x \in \mathbb{R}^{2}: 1 / 4<|x|<1\right\}$ ). In this case we defined in Section 6 the map $\operatorname{deg}(u, \Omega)$ which has all the required properties of $\chi(u)$. Thus, we define $\chi(\cdot):=\operatorname{deg}(\cdot, \Omega): E_{\varepsilon}^{\Lambda} \longrightarrow$ $\mathbb{Z}$. Theorem 9 states that this map is continuous inside each level set of the Ginzburg-Landau energy. Since $\chi$ is a continuous map with values in the discrete set $\mathbb{Z}$, for each $k \in \mathbb{Z}, \chi^{-1}(k)=\left\{u \in E_{\varepsilon}^{\Lambda}: \chi(u)=k\right\}$, will be an open and closed subset of $E_{\varepsilon}^{\Lambda}$ (in $H^{1}$ topology). We have thus succeeded in defining topological sectors inside $E_{\varepsilon}^{\Lambda}$. This concludes the proof of Theorem 1. Theorem 6 follows from Theorem 1 as described in the Introduction.

\section{The Palais-Smale condition: proof of Theorem 3.}

Suppose that $u_{n}$ is a Palais-Smale sequence for $E_{\varepsilon}$, i.e. that there exists a constant $M$ such that

$$
\begin{gathered}
E_{\varepsilon}\left(u_{n}\right) \leq M, \quad \text { for all } n, \\
d E_{\varepsilon}\left(u_{n}\right) \longrightarrow 0 \text { in }\left(H^{1}\right)^{*} \text { as } n \longrightarrow+\infty,
\end{gathered}
$$

where $\left(H^{1}\right)^{*}$ is the dual of $H^{1}\left(\Omega, \mathbb{R}^{2}\right)$, and $d E_{\varepsilon}\left(u_{n}\right)$ denotes the differential of $E_{\varepsilon}$ at $u_{n}$. We want to show that then $u_{n}$ has a strongly convergent subsequence in $H^{1}$. This shall be achieved in two steps: first we prove that $u_{n}$ is bounded in $H^{1}\left(\Omega, \mathbb{R}^{2}\right)$ and then we find a convergent subsequence. 
9.1. Step 1: $u_{n}$ is bounded in $H^{1}$.

Equation (9.1) can be written as

$$
\frac{1}{2} \int_{\Omega}\left|\nabla u_{n}\right|^{2}+\frac{1}{4 \varepsilon^{2}} \int_{\Omega}\left(1-\left|u_{n}\right|^{2}\right)^{2} \leq M, \quad \text { for all } n,
$$

and equation (9.2) means that there is a sequence $C_{n} \geq 0$, such that for all $v \in H^{1}\left(\Omega, \mathbb{R}^{2}\right)$,

$$
\left|\int_{\Omega} \nabla u_{n} \cdot \nabla v-\frac{1}{\varepsilon^{2}} \int_{\Omega}\left(1-\left|u_{n}\right|^{2}\right) u_{n} \cdot v\right| \leq C_{n}\|v\|_{H^{1}\left(\Omega, \mathbb{R}^{2}\right)},
$$

which implies that there exists a sequence $b_{n}(v)$ such that $0 \leq b_{n}(v) \leq$ $C_{n}$, for all $n, v$ (and hence $b_{n} \longrightarrow 0$ ) and

$$
\left|\int_{\Omega} \nabla u_{n} \cdot \nabla v\right|=b_{n}\|v\|_{H^{1}\left(\Omega, \mathbb{R}^{2}\right)}+\left|\frac{1}{\varepsilon^{2}} \int_{\Omega}\left(1-\left|u_{n}\right|^{2}\right) u_{n} \cdot v\right| .
$$

Taking $v=u_{n}$ in (9.4) we obtain

$$
\left.\left|\int_{\Omega}\right| \nabla u_{n}\right|^{2}-\frac{1}{\varepsilon^{2}} \int_{\Omega}\left(1-\left|u_{n}\right|^{2}\right)\left|u_{n}\right|^{2} \mid \leq C_{n}\left\|u_{n}\right\|_{H^{1}\left(\Omega, \mathbb{R}^{2}\right)},
$$

and thus

$$
\left.\left|\int_{\Omega}\right| \nabla u_{n}\right|^{2}\left|\leq C_{n}\left\|u_{n}\right\|_{H^{1}\left(\Omega, \mathbb{R}^{2}\right)}+\right| \frac{1}{\varepsilon^{2}} \int_{\Omega}\left(1-\left|u_{n}\right|^{2}\right)\left|u_{n}\right|^{2} \mid .
$$

First, using the Cauchy-Schwarz inequality and (9.3), we notice that,

$$
\begin{aligned}
\left.\left|\frac{1}{\varepsilon^{2}} \int_{\Omega}\left(1-\left|u_{n}\right|^{2}\right)\right| u_{n}\right|^{2} \mid & =\left|\frac{1}{\varepsilon^{2}} \int_{\Omega}\left(1-\left|u_{n}\right|^{2}\right)^{2}-\frac{1}{\varepsilon^{2}} \int_{\Omega}\left(1-\left|u_{n}\right|^{2}\right)\right| \\
& \leq 4 M+\frac{1}{\varepsilon^{2}}\left(\int_{\Omega}\left(1-\left|u_{n}\right|^{2}\right)^{2}\right)^{1 / 2}|\Omega|^{1 / 2} \\
& \leq 4 M+\frac{2}{\varepsilon} M^{1 / 2}|\Omega|^{1 / 2}
\end{aligned}
$$

Second, the same type of estimate yields

$$
\begin{aligned}
\left.\left|\int_{\Omega}\right| u_{n}\right|^{2} \mid & =\left.\left|\int_{\Omega} 1-\right| u_{n}\right|^{2}+1 \mid \\
& \leq\left.\left|\int_{\Omega} 1-\right| u_{n}\right|^{2}|+| \Omega \mid \\
& \leq 2 M^{1 / 2}|\Omega|^{1 / 2} \varepsilon+|\Omega| \\
& =|\Omega|+o(\varepsilon) .
\end{aligned}
$$


From (9.7) and (9.8) it follows that

$$
\int_{\Omega}\left|\nabla u_{n}\right|^{2} \leq C_{n}\left(\left\|u_{n}\right\|_{L^{2}}+\left\|\nabla u_{n}\right\|_{L^{2}}\right)+4 M+\frac{1}{\varepsilon} 2 M^{1 / 2}|\Omega|^{1 / 2}
$$

and, using (9.9), this yields

$$
\begin{aligned}
\left\|\nabla u_{n}\right\|_{L^{2}}^{2}-C_{n}\left\|\nabla u_{n}\right\|_{L^{2}} \leq & C_{n}\left(2 M^{1 / 2}|\Omega|^{1 / 2} \varepsilon+|\Omega|\right)^{1 / 2} \\
& +4 M+\frac{1}{\varepsilon} 2 M^{1 / 2}|\Omega|^{1 / 2} \\
= & \hat{C}(M, \varepsilon) .
\end{aligned}
$$

Since $C_{n} \longrightarrow 0$ this implies that $\left\|\nabla u_{n}\right\|_{L^{2}(\Omega)}$ is bounded. Together with (9.9), which gives us a bound on $\left\|u_{n}\right\|_{L^{2}(\Omega)}$, this yields

$$
\left\|u_{n}\right\|_{H^{1}(\Omega)} \leq C(M, \varepsilon) \text {. }
$$

which concludes the proof of the first step.

Step 2: $u_{n}$ has a strongly convergent subsequence in $H^{1}$.

Since by $(9.12) u_{n}$ is bounded in $H^{1}\left(\Omega, \mathbb{R}^{2}\right)$, it has a subsequence, which we will still denote by $u_{n}$ which is weakly convergent in $H^{1}\left(\Omega, \mathbb{R}^{2}\right)$. Hence, using the fact that we have a compact embedding $H^{1}\left(\Omega, \mathbb{R}^{2}\right) \hookrightarrow L^{2}(\Omega)$, we know that, up to passing to a subsequence, there exists $u \in H^{1}\left(\Omega, \mathbb{R}^{2}\right)$ such that

$$
u_{n} \longrightarrow u \text { in } L^{2}(\Omega) \text { and } \nabla u_{n} \rightarrow \nabla u \text { in } L^{2}(\Omega) .
$$

Therefore, we just need to prove strong convergence in $L^{2}(\Omega)$ of the gradients, $\nabla u_{n} \longrightarrow \nabla u$ in $L^{2}(\Omega)$. By (9.13) we already have weak convergence $\nabla u_{n} \rightarrow \nabla u$, thus we just need to prove the convergence of the $L^{2}(\Omega)$ norms in order to obtain strong convergence.

Since $H^{1}(\Omega) \hookrightarrow L^{p}(\Omega)$, for all $1 \leq p<+\infty$, we have that

(9.14) $u_{n} \rightarrow u$ in $H^{1}$ implies $u_{n} \longrightarrow u$ in $L^{p}, \quad$ for all $1 \leq p<+\infty$.

In particular

$$
u_{n} \longrightarrow u \text { in } L^{4}(\Omega) \text { and }\left|u_{n}\right|^{2} \longrightarrow|u|^{2} \text { in } L^{4}(\Omega) \text {. }
$$


Thus, using Hölder's inequality,

$$
\begin{gathered}
\left(1-\left|u_{n}\right|^{2}\right) u_{n} \longrightarrow\left(1-|u|^{2}\right) u \text { in } L^{2}(\Omega), \\
\left(1-\left|u_{n}\right|^{2}\right) u_{n} \cdot u \longrightarrow\left(1-|u|^{2}\right)|u|^{2} \text { in } L^{1}(\Omega),
\end{gathered}
$$

and, since $u_{n} \longrightarrow u$ in $L^{2}(\Omega)$,

$$
\left(1-\left|u_{n}\right|^{2}\right) u_{n} \cdot u_{n} \longrightarrow\left(1-|u|^{2}\right)|u|^{2} \text { in } L^{1}(\Omega) .
$$

Taking $v=u \in H^{1}$ in equation (9.5) we obtain

$$
\left|\int_{\Omega} \nabla u_{n} \cdot \nabla u\right|=b_{n}\|u\|_{H^{1}\left(\Omega, \mathbb{R}^{2}\right)}+\left|\frac{1}{\varepsilon^{2}} \int_{\Omega}\left(1-\left|u_{n}\right|^{2}\right) u_{n} \cdot u\right| .
$$

Passing to the limit $n \longrightarrow+\infty$, using the fact that $\nabla u_{n} \rightarrow u$ weakly in $L^{2}(\Omega), b_{n} \longrightarrow 0$ and $(9.15)$, inequality (9.17) yields

$$
\int_{\Omega}|\nabla u|^{2}=\left.\left|\frac{1}{\varepsilon^{2}} \int_{\Omega}\left(1-|u|^{2}\right)\right| u\right|^{2} \mid .
$$

On the other hand, passing to the limit in (9.7), using the fact that $C_{n} \longrightarrow 0,(9.12),(9.16)$ and (9.18), we obtain

$$
\lim _{n \rightarrow+\infty} \int_{\Omega}\left|\nabla u_{n}\right|^{2} \leq\left.\left.\left|\frac{1}{\varepsilon^{2}} \int_{\Omega}\left(1-|u|^{2}\right)\right| u\right|^{2}\left|=\int_{\Omega}\right| \nabla u\right|^{2} .
$$

Since by the lower semi-continuity of the $L^{2}$ norm in weak topology we have that

$$
\int_{\Omega}|\nabla u|^{2} \leq \lim _{n \rightarrow+\infty} \int_{\Omega}\left|\nabla u_{n}\right|^{2}
$$

equation (9.19) implies that

$$
\int_{\Omega}|\nabla u|^{2}=\lim _{n \rightarrow+\infty} \int_{\Omega}\left|\nabla u_{n}\right|^{2}
$$

which concludes the proof of Theorem 3 for $E_{\varepsilon}$. For the case of the functional $F_{\varepsilon}$ the same proof will work once we fix the Coulomb gauge. The reader interested in seeing how the gauge invariance affects PalaisSmale sequences in this problem may take a look at the appendix of $[4]$. 


\section{Threshold energies and components of $E_{\varepsilon}^{\Lambda}$.}

We can reformulate the statement of Theorem 4 and state the following Proposition.

Proposition 2. Suppose that for some $\Lambda \in \mathbb{R}^{+}$, we have that for some $\varepsilon<\varepsilon_{0}$ (where $\varepsilon_{0}$ is given Theorem 1 ) there exist $n, k \in \mathbb{Z}, n \neq k$, such that the topological sectors $\operatorname{top}_{n}\left(E_{\varepsilon}^{\Lambda}\right)$ and $\operatorname{top}_{k}\left(E_{\varepsilon}^{\Lambda}\right)$ are both nonempty. Then, there are mountain-pass type critical points of $E_{\varepsilon}$ or, equivalently, there exist mountain-pass type solutions of the GinzburgLandau equations (1.11).

More precisely, consider two non-empty components of $E_{\varepsilon}^{\Lambda}, \Sigma_{0} \subset$ $\operatorname{top}_{n}\left(E_{\varepsilon}^{\Lambda}\right)$ and $\Sigma_{1} \subset \operatorname{top}_{k}\left(E_{\varepsilon}^{\Lambda}\right)$, and let $c_{n, k}\left(\Sigma_{0}, \Sigma_{1}\right)$ be defined as in (10.4). Then, there exists a map $u \in H^{1}\left(\Omega, \mathbb{R}^{2}\right)$ which is a critical point of $E_{\varepsilon}$ and such that $E_{\varepsilon}(u)=c_{n, k}\left(\Sigma_{0}, \Sigma_{1}\right)$.

Since $H^{1}(\Omega)$ is locally pathwise connected and the level sets $E_{\varepsilon}^{\Lambda}$ are open, their path components coincide with their components, so we can use the two concepts indistinguishably. Let $n, k \in \mathbb{Z}$ be two distinct integers, and let $\Sigma_{0}$ and $\Sigma_{1}$ be components of $E_{\varepsilon}^{\Lambda}$ such that $\Sigma_{0} \subset \operatorname{top}_{n}\left(E_{\varepsilon}^{\Lambda}\right)$ and $\Sigma_{1} \subset \operatorname{top}_{k}\left(E_{\varepsilon}^{\Lambda}\right)$. Then, given $u_{0}, u_{0}^{\prime} \in \Sigma_{0}$ and $u_{1}, u_{1}^{\prime} \in \Sigma_{1}$, we know that there exist two paths $\gamma_{i}, i=0,1$, such that

$$
\gamma_{i}:[0,1] \longrightarrow \Sigma_{i}, \gamma_{i}(0)=u_{i}, \gamma_{i}(1)=u_{i}^{\prime}, i=0,1
$$

In particular,

$$
\gamma_{i}(s)<\Lambda, \quad \text { for all } s \in[0,1]
$$

As usual, we define the composition operation for paths: let $\gamma$ be a path from $p$ to $q$, and $\sigma$ be a path from $q$ to $r$, then $\varrho=\gamma \sigma$ is the path from $p$ to $r$ defined by

$$
\varrho(s):= \begin{cases}\gamma(2 s), & \text { for } 0 \leq s \leq \frac{1}{2} \\ \sigma(2 s-1), & \text { for } \frac{1}{2} \leq s \leq 1 .\end{cases}
$$

And we define the inverse path of $\gamma$, which we denote by $\gamma^{-1}$, as $\gamma^{-1}(s):=\gamma(1-s)$, for $s \in[0,1]$. Then, to any path $\gamma:[0,1] \longrightarrow H^{1}(\Omega)$ between $u_{0}$ and $u_{1}$, one can associate a path $\gamma^{\prime}=\gamma_{0}^{-1} \gamma \gamma_{1}:[0,1] \longrightarrow$ 
$H^{1}(\Omega)$ from $u_{0}^{\prime}$ to $u_{1}^{\prime}$. And vice-versa, to any path $\gamma^{\prime}:[0,1] \longrightarrow H^{1}(\Omega)$ between $u_{0}^{\prime}$ and $u_{1}^{\prime}$, one can associate a path $\gamma=\gamma_{0} \gamma^{\prime} \gamma_{1}^{-1}:[0,1] \longrightarrow$ $H^{1}(\Omega)$ from $u_{0}$ to $u_{1}$. With these definitions, from equation (10.1) it follows that

$$
\max _{s \in[0,1]} E_{\varepsilon}(\gamma(s))=\max _{s \in[0,1]} E_{\varepsilon}\left(\gamma^{\prime}(s)\right) \geq \Lambda
$$

And hence,

$$
\inf _{\gamma \in \mathcal{V}}\left(\max _{s \in[0,1]}\left(E_{\varepsilon}(\gamma(s))\right)\right)=\inf _{\gamma \in \mathcal{V}^{\prime}}\left(\max _{s \in[0,1]}\left(E_{\varepsilon}\left(\gamma^{\prime}(s)\right)\right)\right) \geq \Lambda
$$

where,

$$
\mathcal{V}:=\left\{\gamma \in C^{0}\left([0,1], H^{1}\left(\Omega, \mathbb{R}^{2}\right)\right): \gamma(0)=u_{0}, \text { and } \gamma(1)=u_{1}\right\},
$$

and

$$
\mathcal{V}^{\prime}:=\left\{\gamma^{\prime} \in C^{0}\left([0,1], H^{1}\left(\Omega, \mathbb{R}^{2}\right)\right): \gamma^{\prime}(0)=u_{0}^{\prime}, \text { and } \gamma^{\prime}(1)=u_{1}^{\prime}\right\} .
$$

Thus, $c_{n}$, the threshold energy for a transition from $u_{0}$ to $u_{1}$ defined in (1.16), is well defined as a transition energy from a component $\Sigma_{0}$ of $\operatorname{top}_{n}\left(E_{\varepsilon}^{\Lambda}\right)$ to a component $\Sigma_{1}$ of top $k\left(E_{\varepsilon}^{\Lambda}\right)$. We can define,

$$
c_{n, k}\left(\Sigma_{0}, \Sigma_{1}\right):=\inf _{\gamma \in \mathcal{V}_{n, k}\left(\Sigma_{0}, \Sigma_{1}\right)}\left(\max _{s \in[0,1]}\left(E_{\varepsilon}(\gamma(s))\right)\right)
$$

where,

$$
\begin{aligned}
\mathcal{V}_{n, k}\left(\Sigma_{0}, \Sigma_{1}\right) & \\
\quad:=\left\{\gamma \in C^{0}\left([0,1], H^{1}\left(\Omega, \mathbb{R}^{2}\right)\right):\right. & \gamma(0) \in \Sigma_{0} \subset \operatorname{top}_{n}\left(E_{\varepsilon}^{\Lambda}\right), \\
& \text { and } \left.\gamma(1) \in \Sigma_{1} \subset \operatorname{top}_{k}\left(E_{\varepsilon}^{\Lambda}\right)\right\} .
\end{aligned}
$$

By the Mountain Pass Theorem we know that $c_{n, k}\left(\Sigma_{0}, \Sigma_{1}\right)$ is a generalized critical value of $E_{\varepsilon}$ and, since by Theorem 3 the functional $E_{\varepsilon}$ satisfies the Palais-Smale condition, this implies that $c_{n, k}\left(\Sigma_{0}, \Sigma_{1}\right)$ is also a critical value of $E_{\varepsilon}$, thus concluding the proof of Proposition 2 and Theorem 4.

REMARK. For small $\varepsilon$ and $n \neq k, c_{n, k}\left(\Sigma_{0}, \Sigma_{1}\right)$ shouldn't depend on the specific components $\Sigma_{0} \subset \operatorname{top}_{n}\left(E_{\varepsilon}^{\Lambda}\right)$ and $\Sigma_{1} \subset \operatorname{top}_{k}\left(E_{\varepsilon}^{\Lambda}\right)$, but only on $n$ and $k$ (i.e. only on the topological sectors themselves). This leads 
us back to the question of how many distinct components can there be inside a topological sector and how do they change when $\Lambda$ changes. We expect that for certain values of $\Lambda, \operatorname{top}_{n}\left(E_{\varepsilon}^{\Lambda}\right)$ may not be connected, but that as we increase $\Lambda$ the different components which existed at lower energies, should increase in size and eventually intersect thus becoming the same component. As a matter of fact, in [1] we will be able to prove that all the components in $\operatorname{top}_{n}\left(E_{\varepsilon}^{\Lambda}\right)$ can be connected by paths wich involve energies of, at most, something like $6 \Lambda$, while to connect different topological sectors we will need energies like $\pi|\log \varepsilon|$, which for small enough $\varepsilon$ is much bigger than $6 \Lambda$. In this case $c_{n, k}\left(\Sigma_{0}, \Sigma_{1}\right)$ will depend only on $n$ and $k$ as we said.

REMARK. As usual, similar results are valid for $F_{\varepsilon}$.

\section{A model for superconductivity.}

In this section we will consider the gauge-invariant Ginzburg-Landau model (1.3), and prove that inside the level sets $F_{\varepsilon}^{\Lambda}$ we can define topological sectors in a similar way to the one used for defining such sectors inside the level sets $E_{\varepsilon}^{\Lambda}$ in theorems 1 and 6 which we proved in Section 8.

\subsection{Gauge fixing.}

Given a configuration $(v, B) \in F_{\varepsilon}^{\Lambda}$, we will show in this section how to choose a gauge equivalent configuration, $(u, A) \approx(v, B)$, such that we have the necessary control on $A$ to allow us to bound the $L^{2}$ norm of $\nabla u$ by a constant depending only on the energy level $\Lambda$. In fact, to achieve this, all we need to do is to fix a Coulomb gauge over the unit disk $D=B(0,1)=\Omega \cup B(0,1 / 4)$.

Proposition 3. Given a configuration $(v, B) \in H^{1}$, there exists $(u, A) \approx$ $(v, B)$ such that

$$
\left\{\begin{array}{l}
d^{\star} A=0, \quad \text { in } D, \\
A \cdot \nu=0, \quad \text { on } \partial D=S^{1} .
\end{array}\right.
$$


The proof is just the same as that of [9, Propositions I.1 and I.2]. Now we remark that, since $D$ is simply-connected, (11.1) implies that there exists $\zeta \in H^{2}(D, \mathbb{R})$ such that writing $\hat{\zeta}=\zeta d x^{1} \wedge d x^{2}=\star \zeta$,

$$
\begin{cases}A=d^{\star} \hat{\zeta}=\star d \zeta, & \text { in } D, \\ \zeta=0, & \text { on } \partial D .\end{cases}
$$

It follows from (11.1) and (11.2) that $\zeta$ satisfies

$$
\begin{cases}\Delta \zeta=d^{\star} d \zeta=\star d A, & \text { in } D, \\ \zeta=0, & \text { on } \partial D .\end{cases}
$$

This implies, using standard elliptic estimates, that

$$
\|\zeta\|_{W^{2,2}(D)} \leq \hat{C}\|d A\|_{L^{2}(D)},
$$

which, together with (11.2) yields

$$
\begin{aligned}
\|A\|_{W^{1,2}(D)}^{2} & =\int_{D}|A|^{2}+\int_{D}|\nabla A|^{2} \\
& =\int_{D}|\nabla \zeta|^{2}+\int_{D}\left|\nabla^{2} \zeta\right|^{2} \\
& \leq\|\zeta\|_{W^{2,2}(D)}^{2} \\
& \leq \hat{C}\|d A\|_{L^{2}(D)}^{2} \\
& \leq \hat{C} F_{\varepsilon}(u, A) \\
& <\hat{C} \Lambda
\end{aligned}
$$

where $\hat{C}$ is a constant.

\subsection{Global control of $|\nabla u|^{2}$.}

The purpose of this subsection is to show how to obtain a bound on $\|\nabla u\|_{L^{2}(\Omega)}$ by a constant depending only on the energy level $\Lambda$.

Lemma 6. Given $(v, B) \in F_{\varepsilon}^{\Lambda}$, let $(u, A)$ be as in Proposition 3. Then,

$$
\int_{\Omega}|\nabla u|^{2} \leq C
$$


where $C$ is a constant which only depends on $\Lambda$.

Proof. Since, by construction, $F_{\varepsilon}(u, A)=F_{\varepsilon}(v, B) \leq \Lambda$, we have that, in particular,

$$
\int_{\Omega}\left|\nabla_{A} u\right|^{2}=\int_{\Omega}|\nabla u-\imath A u|^{2} \leq 2 F_{\varepsilon}(u, A) \leq 2 \Lambda .
$$

Hence,

$$
\begin{aligned}
\int_{\Omega}|\nabla u|^{2} & =\int_{\Omega}|\nabla u-\imath A u+\imath A u|^{2} \\
& \leq 2 \int_{\Omega}|\nabla u-\imath A u|^{2}+2 \int_{\Omega}|A u|^{2} \\
& \leq 4 F_{\varepsilon}(u, A)+2 \int_{\Omega}|A|^{2}|u|^{2} \\
& \leq 4 \Lambda+2 \int_{\Omega}|A|^{2}\left(|u|^{2}-1\right)+2 \int_{\Omega}|A|^{2} \\
& \leq 4 \Lambda+\left.\left.2 \int_{\Omega}|A|^{2}|1-| u\right|^{2}\left|+2 \int_{\Omega}\right| A\right|^{2} .
\end{aligned}
$$

Using Hölder's inequality, and the fact that from the energy bound it follows that

$$
\left\|1-|u|^{2}\right\|_{L^{2}(\Omega)}^{2} \leq 4 \varepsilon^{2} F_{\varepsilon}(u, A) \leq 4 \varepsilon^{2} \Lambda,
$$

we obtain

$$
\begin{aligned}
\int_{\Omega}|\nabla u|^{2} & \leq 4 \Lambda+2\left\|A^{2}\right\|_{L^{2}(\Omega)}\left\|1-|u|^{2}\right\|_{L^{2}(\Omega)}+2\|A\|_{L^{2}(\Omega)}^{2} \\
& \leq 4 \Lambda+4 \varepsilon \Lambda^{1 / 2}\|A\|_{L^{4}(\Omega)}^{2}+2\|A\|_{L^{2}(\Omega)}^{2} .
\end{aligned}
$$

Since we are in a two-dimensional domain it follows from the Sobolev Embedding Theorem that $W^{1,2}(\Omega) \hookrightarrow L^{q}(\Omega)$, for all $q<+\infty$. hence, in particular, there exists a constant $\tilde{C}$ such that

$$
\|A\|_{L^{4}(\Omega)} \leq \tilde{C}\|A\|_{W^{1,2}(\Omega)} \cdot
$$

Furthermore, from (11.4) we know that

$$
\|A\|_{W^{1,2}(\Omega)} \leq\|A\|_{W^{1,2}(D)} \leq \sqrt{\hat{C} \Lambda} .
$$


From equations (11.8), (11.9) and (11.10) it follows that for $\varepsilon<1$ (as mentioned before, it is the case where $\varepsilon$ is small that interests us),

$$
\begin{aligned}
\int_{\Omega}|\nabla u|^{2} & \leq 4 \Lambda+4 \varepsilon \Lambda^{1 / 2} \tilde{C}^{2}\|A\|_{W^{1,2}(D)}^{2}+2\|A\|_{W^{1,2}(D)}^{2} \\
& \leq 4 \Lambda+4 \Lambda^{1 / 2} \tilde{C}^{2} \hat{C} \Lambda+2 \Lambda \hat{C}=C
\end{aligned}
$$

where $C$ is a constant depending only on $\Lambda$.

\subsection{Definition of $\operatorname{deg}([v, B], \Omega)$ and proof of Theorem 2 .}

Once we have the estimate (11.5), we can define $\operatorname{deg}(u, \Omega)$ as in the case of the initial model (1.1), since we will have all the estimates we used in the work that culminated with the definition of the degree in Section 6. Thus, for $\varepsilon$ sufficiently small, $\operatorname{deg}(u, \Omega)$ is well defined, and hence we may define

$$
\operatorname{deg}([v, B], \Omega):=\operatorname{deg}(u, \Omega) .
$$

Once we have achieved this, Theorem 2 follows from the corresponding result for $\operatorname{deg}(u, \Omega)$ which, thanks to estimate (11.5), can be proven in a similar way to that we used for proving Theorem 1 (therefore, we omit this proof).

The generalization of Theorem 2 to the setting of Riemannian manifolds will then follow from Theorem 2 in an analogous way as Theorem 6 followed from Theorem 1.

\section{Appendix: Covering Lemma.}

This section is devoted to a general covering Lemma we used to prove Lemma 4.

Lemma 7. Let $\varepsilon>0$ and $W_{1}, \ldots, W_{n}$ be connected open subsets of $\mathbb{R}^{2}$ such that there exist $C, \alpha>0$ such that $\operatorname{diam}\left(W_{l}\right) \leq C \varepsilon^{\alpha}$. Then, for $\varepsilon$ sufficiently small, there is a family of numbers $\alpha_{1}, \ldots, \alpha_{m} \geq \alpha / 2$, and a family of balls $B_{1}, \ldots, B_{m}$, with $m \leq n$, such that, denoting by $x_{j}$ the center of $B_{j}$, and by $r_{j}$ its radius,

i) $r_{j} \leq C \varepsilon^{\alpha_{j}}$. 
ii) $\bigcup_{l=1}^{n} W_{l} \subset \bigcup_{j=1}^{m} B_{j}$.

iii) The enlarged balls $\tilde{B}_{j}:=B\left(x_{j}, \varepsilon^{-\alpha_{j} /\left(2^{n+1}+1\right)} r_{j}\right)$ are pairwise disjoint.

ProOF. We start by defining

$$
\begin{gathered}
q_{n}:=\frac{2^{n+1}}{2^{n+1}+1}, \\
p_{k}:=\frac{1}{\sum_{j=0}^{k} 2^{-j}}=\frac{2^{k}}{2^{k+1}-1},
\end{gathered}
$$

for $k=1, \ldots, n$.

The proof of this Lemma is done by induction on the number $k$ of components of $A=\bigcup_{l=1}^{n} W_{l}$. For $k=1$, it suffices to consider a unique ball of radius $r_{1}=C \varepsilon^{\alpha_{1}}$, with $\alpha_{1}=2 \alpha / 3=\alpha p_{1}$, since, for $\varepsilon$ sufficiently small,

$$
\operatorname{diam}(A) \leq \sum_{l=1}^{n} \operatorname{diam}\left(W_{l}\right) \leq n C \varepsilon^{\alpha} \leq C \varepsilon^{2 \alpha / 3} .
$$

Hence, we can find a ball $B_{1}$, of radius $r_{1} \leq C \varepsilon^{2 \alpha / 3}$ containing $\bigcup_{l=1}^{n} W_{l}$.

Suppose that the result is always true if $A$ has $\bar{n}$ components, for all $\bar{n} \leq k-1 \leq n-1$, and, furthermore, the number $m$ of balls obtained in the covering process is at most $\bar{n}$ and each of the $\alpha_{j}$ 's obtained satisfies

$$
\alpha_{j} \geq \frac{\alpha}{\sum_{j=0}^{\bar{n}} 2^{-j}}=\alpha p_{\bar{n}} \geq \alpha p_{k-1} .
$$

To complete the induction argument, we just have to show that then the result will still be true when $\mathrm{A}$ has $k$ components, and that in this case $m \leq k \leq n$ and we can find $\alpha_{j}$ 's such that

$$
\alpha_{j} \geq \frac{\alpha}{\sum_{j=0}^{k} 2^{-j}}=\alpha p_{k}
$$


Let $A_{1}, \ldots, A_{k}$ be the connected components of $A$. Suppose that

$$
\operatorname{diam}(A) \leq 5 n C \varepsilon^{\alpha q_{n} p_{k-1}} .
$$

Then, for $\varepsilon$ sufficiently small, we can include $A$ in a ball $B_{1}$ of radius $r_{1} \leq \varepsilon^{\alpha p_{k}}$. In fact, it suffices that

$$
3 n C \varepsilon^{\alpha q_{n} p_{k-1}} \leq C \varepsilon^{\alpha q_{n} p_{k}} .
$$

This is always true, provided that $\varepsilon$ is sufficiently small, since

$$
\alpha q_{n} p_{k-1}>\alpha p_{k} \quad \text { if and only if } \quad \frac{p_{k-1}}{p_{k}}>\frac{1}{q_{n}}
$$

and

$$
\frac{p_{k-1}}{p_{k}}=1+\frac{2^{-k}}{p_{k-1}}>1+\frac{1}{2^{k+1}}=\frac{2^{k+1}}{2^{k+1}+1} \geq \frac{2^{n+1}}{2^{n+1}+1}=\frac{1}{q_{n}} .
$$

Thus, if (12.3) is true, our proof will be completed. Hence, we may suppose that this is not so, i.e., that

$$
\operatorname{diam}(A) \geq 5 n C \varepsilon^{\alpha q_{n} p_{k-1}} .
$$

Let $y_{1}, y_{2} \in \bar{A}$ be such that $\left|y_{1}-y_{2}\right|=\operatorname{diam}(A)$, and consider the family of balls $B\left(y_{1}, r\right)$ for $r \in(0, \operatorname{diam}(A))$. Define $G_{j}:=\left\{r: B\left(y_{1}, r\right) \cap A_{j} \neq\right.$ $\varnothing\}, j=1, \ldots, k$. Each $G_{j}$ will be an interval, and the sum of the lengths of the $G_{j}$ 's will be smaller than the sum of the diameters of the $W_{l}$ 's, which is at most $n C \varepsilon^{\alpha}$. Since $n C \varepsilon^{\alpha} \leq n C \varepsilon^{\alpha q_{n} p_{k-1}}$, for all $\varepsilon \leq 1$, it follows that the set

$$
\hat{G}:=(0, \operatorname{diam}(A)) \backslash \bigcup_{j=1}^{k} G_{j}
$$

will have a measure of at least

$$
5 n C \varepsilon^{\alpha q_{n} p_{k-1}}-n C \varepsilon^{\alpha q_{n} p_{k-1}}=4 n C \varepsilon^{\alpha q_{n} p_{k-1}} .
$$

Moreover, the set $\hat{G}$ is the union of, at most, $k-1$ subintervals of $(0, \operatorname{diam}(A))$ since it was obtained from the latter by removing the $k$ open intervals $G_{j}$ (among which one had endpoint 0 and another 
had endpoint diam $(A)$ ). Consequently, at least one of its components, which we will denote by $\left[a_{0}, b_{0}\right]$, will be such that

$$
b_{0}-a_{0} \geq \frac{|\hat{G}|}{k-1} \geq \frac{4 n}{k-1} \varepsilon^{\alpha q_{n} p_{k-1}}>4 \varepsilon^{\alpha q_{n} p_{k-1}} .
$$

Let $\hat{A}=A \cap B\left(y_{1}, a_{0}\right)$, and $\tilde{A}=A \backslash B\left(y_{1}, b_{0}\right)$. Then, $A=\hat{A} \cup \tilde{A}$, and both $\hat{A}$ and $\tilde{A}$ include at least one of the $A_{j}$ 's. Hence, both $\hat{A}$ and $\tilde{A}$ have at most $k-1$ components and thus we can apply the induction step to each of them. It yields, since the sum of the number of components of $\hat{A}$ and $\tilde{A}$ is $k$, that there will be a total of $m \leq k$ balls $B_{1}, \ldots, B_{m}$, such that

a) $\hat{A} \subset B_{1} \cup \cdots \cup B_{\bar{m}}, \tilde{A} \subset B_{\bar{m}+1} \cup \cdots \cup B_{m}$, for some $\bar{m}<m$.

b) Each $B_{j}$ has center $x_{j}$ and radius $r_{j} \leq C \varepsilon^{\alpha_{j}}$, where $\alpha_{j} \geq$ $\alpha p_{k-1} \geq \alpha p_{k}$.

c) The enlarged balls $\tilde{B}_{j}:=B\left(x_{j}, \varepsilon^{-\alpha_{j} /\left(2^{n+1}+1\right)} r_{j}\right)$ are pairwise disjoint for $j \in\{1, \ldots, \bar{m}\}$ and also for $j \in\{\bar{m}+1, \ldots, m\}$.

However, to obtain the disjointness of two $\tilde{B}_{j}$, one corresponding to $\hat{A}$ (i.e. $j \leq \bar{m}$ ) and the other to $\tilde{A}$ (i.e. $j>\bar{m}$ ), we need to use equation (12.5). In fact, if $j_{1} \leq \bar{m}$ and $j_{2}>\bar{m}$, then

$$
\left|x_{j_{1}}-y_{1}\right|<a_{0}+C \varepsilon^{\alpha_{j_{1}}}<a_{0}+C \varepsilon^{\alpha q_{n} p_{k-1}},
$$

since $B_{j_{1}} \cap \hat{A} \neq \varnothing, \hat{A} \subset B\left(y_{1}, a_{0}\right)$ and by b), $\alpha_{j_{1}} \geq \alpha p_{k-1}>q_{n} \alpha p_{k-1}$. Similarly, we have that

$$
\left|x_{j_{2}}-y_{1}\right|>b_{0}-C \varepsilon^{\alpha_{j_{2}}}>b_{0}-C \varepsilon^{\alpha q_{n} p_{k-1}},
$$

since $B_{j_{2}} \cap \tilde{A} \neq \varnothing, \tilde{A} \subset A \backslash B\left(y_{1}, b_{0}\right)$ and, by b), $\alpha_{j_{2}} \geq \alpha p_{k-1}>$ $q_{n} \alpha p_{k-1}$.

Therefore, combining (12.6) and (12.7) we have

$$
\left|x_{j_{1}}-x_{j_{2}}\right|>2 C \varepsilon^{\alpha q_{n} p_{k-1}} .
$$

Since $\tilde{B}_{j_{i}}$ has radius

$$
C \varepsilon^{q_{n} \alpha_{j_{i}}}<C \varepsilon^{\alpha q_{n} p_{k-1}},
$$

equation (12.8) implies that

$$
\tilde{B}_{j_{1}} \cap \tilde{B}_{j_{2}}=\varnothing,
$$


as desired. Consequently, the balls $B_{j}$ obtained satisfy all the conditions required for the induction argument, and thus the proof of Lemma 7 in completed.

REMARK. Relative to the similar covering argument of Lin [22], our result has the advantage that we are able to keep the $\alpha_{j}$ always bigger than $\alpha / 2$, which corresponds to keeping the balls $B_{j}$ rather small - in Lin's result $\alpha_{j}$ may tend to zero when $n \longrightarrow \infty$. However, we also lose something, both because our proof is technically more complicated, but also because we obtain smaller (and more complex) expansion factors for the $\tilde{B}_{j}$ 's. In fact, even Lin's expansion factors $\left(\varepsilon^{-\alpha_{j} / 3}\right)$ go to 1 when $n \longrightarrow \infty$, but ours $\left(\varepsilon^{-\alpha_{j} /\left(2^{n+1}+1\right)}\right)$ will decrease to 1 considerably faster.

We prefered to privilege the scale of the balls because it enables us to assert that in our problem, at least at a scale $\varepsilon^{1 / 2}$, things appear neutral to an outside observer (and it also makes the energy explosion estimate (5.13) slightly neater). Using Lin's result, the scale would depend on $\mathrm{n}$, and hence on $\Lambda$, which would be less satisfactory.

Aknowledgements. A preliminary version of this work was included in the author's thesis done under the supervision of F. Bethuel and presented at the ENS Cachan in January 1996. The author would like to thank him for many fruitful discussions.

\section{References.}

[1] Almeida, L., Transition energies for Ginzburg-Landau functionals. To appear in Nonlinearity.

[2] Almeida, L., Bethuel, F., Méthodes topologiques pour l'équation de Ginzburg-Landau. C. R. Acad. Sci. Paris 320 Série I (1995), 935-938.

[3] Almeida, L., Bethuel, F., Topological methods for the Ginzburg-Landau equation. J. Math. Pures et Appl. 77 (1998), 1-49.

[4] Almeida, L., Bethuel, F., Multiplicity results for the Ginzburg-Landau equation in the presence of symmetries. Houston J. Math. 23 (1997), 733-764.

[5] Almeida, L., Bethuel, F., Guo, Y., A remark on instability of the symmetric vortices with large charge and coupling constant. Comm. Pure Appl. Math. 50 (1997), 1295-1300. 
[6] Bethuel, F., The approximation problem for Sobolev maps between manifolds. Acta Math. 167 (1991), 153-206.

[7] Bethuel, F., Brezis, H., Hélein, F., Asymptotics for the minimization of a Ginzburg-Landau functional. Calc. Var. and Partial Diff. Equations 1 (1993), 123-148.

[8] Bethuel, F., Brezis, H., Hélein, F., Ginzburg-Landau vortices. Birkhäuser, 1994.

[9] Bethuel, F., Rivière, T., A minimization problem related to superconductivity. Ann. Inst. H. Poincaré, Analyse non linéaire 12 (1995), 243-303.

[10] Bethuel, F., Rivière, T., Vorticité dans les modèles de Ginzburg-Landau pour la supraconductivité. Actes du Séminaire EDP 1993-1994. Ecole Polytechnique. Exposé XVI.

[11] Boutet de Monvel-Berthier, A., Georgescu, V., Purice, R., A boundary value problem related to the Ginzburg-Landau model. Comm. Math. Phys. 142 (1991), 1-23.

[12] Demoulini, S., Stuart, D., Gradient flow of the superconducting Ginzburg-Landau functional on the plane. Comm. Math. Phys. 5 (1997), 121-198.

[13] Evans, L., Gariepy, R., Measure theory and fine properties of functions. CRC Press, 1992.

[14] Gilbarg, D., Trudinger, N., Elliptic partial differential equations of second order. 2nd edition, Grundlehren Math. Wiss. 224, Springer, 1983.

[15] Guo, Y., Instability of the symmetric vortices with large charge and coupling constant. Comm. Pure Appl. Math. 69 (1996), 1051-1080.

[16] Jimbo, S., Morita, Y., Ginzburg-Landau equation and stable solutions in a rotational domain. SIAM J. Math. Anal. 27 (1996), 1360-1385.

[17] Jimbo, S., Morita, Y., Stable solutions with zeros to the GinzburgLandau equation with Neumann boundary condition. J. Diff. Equations (1996), 596-613.

[18] Jimbo, S., Morita, Y., Zhai, J., Ginzburg-Landau equation and stable steady state solutions in a non-trivial domain. Comm. Partial Diff. Equations 20 (1995), 2093-2112.

[19] Jimbo, S., Zhai, J., Ginzburg-Landau equation with magnetic effect: non-simply-connected domains. Preprint Univ. Hokkaido 303.

[20] Jaffe, A., Taubes, C., Vortices and monopoles. Birkhäuser, 1980.

[21] Lin, F. H., Some dynamical properties of Ginzburg-Landau vortices. Ann. Inst. H. Poincaré, Analyse non linéaire 12 (1995), 599-622. 
[22] Lin, F. H., Solutions of Ginzburg-Landau equations and critical points of the renormalized energy. Ann. Inst. H. Poincaré, Analyse non linéaire 12 (1995), 599-622.

[23] Rivière, T., Lignes de tourbillon dans le modèle abélien de Higgs. C. R. Acad. Sci. Paris 321 Série I (1995), 73-76.

[24] Rivière, T., Line vortices in the $U(1)$ Higgs model. ESAIM: COCV 1 (1996), 77-167.

[25] Rubinstein, J., Sternberg, P., Homotopy classification of minimizers of the Ginzburg-Landau energy and the existence of permanent currents. Comm. Math. Phys. 179 (1996), 257-263.

[26] Saint-James, D., Sarma, G., Thomas, E. J., Type II superconductivity. Pergamon Press, 1969.

[27] Tilley, D., Tilley, T., Superfluidity and superconductivity. Adam Hilger Ltd., 1990.

[28] White, B., Homotopy classes in Sobolev spaces and the existence of energy minimizing maps. Acta Math. 160 (1988), 1-17.

Recibido: 25 de noviembre de 1.997

Luís Almeida

Centre de Mathématiques et de Leurs Applications

Unité associée au CNRS URA-1611

Ecole Normale Supérieure de Cachan

61 Avenue du Président Wilson

94235 CACHAN Cedex, FRANCE

Luis . Almeida@cmla.ens-cachan.fr 